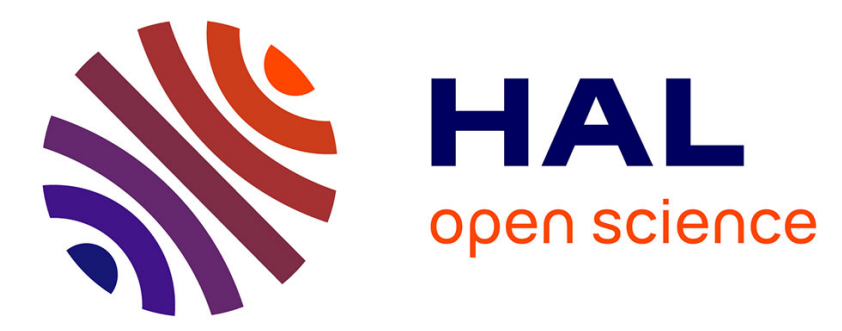

\title{
Macroscopic momentum and mechanical energy equations for incompressible single-phase flow in porous media
}

Catherine Teresa Paéz-García, Francisco J Valdés-Parada, Didier Lasseux

\section{To cite this version:}

Catherine Teresa Paéz-García, Francisco J Valdés-Parada, Didier Lasseux. Macroscopic momentum and mechanical energy equations for incompressible single-phase flow in porous media. Physical Review E , 2017, 95, 10.1103/physreve.95.023101 . hal-03140897

\section{HAL Id: hal-03140897 \\ https://hal.science/hal-03140897}

Submitted on 14 Feb 2021

HAL is a multi-disciplinary open access archive for the deposit and dissemination of scientific research documents, whether they are published or not. The documents may come from teaching and research institutions in France or abroad, or from public or private research centers.
L'archive ouverte pluridisciplinaire HAL, est destinée au dépôt et à la diffusion de documents scientifiques de niveau recherche, publiés ou non, émanant des établissements d'enseignement et de recherche français ou étrangers, des laboratoires publics ou privés. 


\title{
Macroscopic momentum and mechanical energy equations for incompressible single-phase flow in porous media
}

\author{
Catherine Teresa Paéz-García and Francisco J. Valdés-Parada \\ Departamento de I.P.H., Universidad Autónoma Metropolitana-Iztapalapa. Av. San Rafael Atlixco 186, Col. Vicentina, CDMX, 09340, Mexico \\ Didier Lasseux ${ }^{*}$ \\ CNRS, UMR 5295, Univ. Bordeaux, Esplanade des Arts et Métiers, 33405 Talence, Cedex, France
}

\begin{abstract}
Modeling flow in porous media is usually focused on the governing equations for mass and momentum transport, which yield the velocity and pressure at the pore or Darcy scales. However, in many applications, it is important to determine the work (or power) needed to induce flow in porous media, and this can be achieved when the mechanical energy equation is taken into account. At the macroscopic scale, this equation may be postulated to be the result of the inner product of Darcy's law and the seepage velocity. However, near the porous medium boundaries, this postulate seems questionable due to the spatial variations of the effective properties (velocity, permeability, porosity, etc.). In this work we derive the macroscopic mechanical energy equation using the method of volume averaging for the simple case of incompressible single-phase flow in porous media. Our analysis shows that the result of averaging the pore-scale version of the mechanical energy equation at the Darcy scale is not, in general, the expected product of Darcy's law and the seepage velocity. As a matter of fact, this result is only applicable in the bulk region of the porous medium and, in the derivation of this result, the properties of the permeability tensor are determinant. Furthermore, near the porous medium boundaries, a more novel version of the mechanical energy equation is obtained, which incorporates additional terms that take into account the rapid variations of structural properties taking place in this particular portion of the system. This analysis can be applied to multiphase and compressible flows in porous media and in many other multiscale systems.
\end{abstract}

DOI: 10.1103/PhysRevE.95.023101

\section{INTRODUCTION}

The mechanical energy equation is, together with the total mass and momentum equations, a key relationship for the study of fluid dynamics in many systems. As a matter of fact, the study of many engineering systems requires the use of the macroscopic versions of these three equations [see Chapter 7 in Ref. [1]]. In the particular case of the macroscopic mechanical energy equation, a challenge usually lies on the prediction of the rate at which mechanical energy decreases in a system due to viscous dissipation. Interestingly, in natural porous systems, such as geological media, the information provided by the mechanical energy equation is usually not crucial, as, for instance, in the macroscopic study of groundwater flow. Therefore, the focus is directed to the macroscopic conservation equations of mass and momentum, i.e., the continuity equation and Darcy's law. This is due to the interest on the prediction of the piezometric head, which can be experimentally measured [see Chapter 4 in [2]]. However, as emphasized by Celia and Norbotten [3] in engineering systems such as geothermal engineering, enhanced oil recovery, or geological $\mathrm{CO}_{2}$ sequestration, the use of the continuity equation and Darcy's law is insufficient and it is necessary to take into account the information provided by the macroscopic mechanical energy equation, which is related to the total head [see Eq. 4.1.5 in Ref. [2]]. In addition, in applications such as the disposal of high-level radioactive wastes, understanding of the thermohydromechanical processes taking place in the porous medium is crucial as remarked by Chen et al. [4].

*didier.lasseux@u-bordeaux.fr
Furthermore, the study of the viscous dissipation term in the mechanical energy equation is also relevant in the study of nanofibrous media, in specific when the goal is to achieve outstanding properties such as stiffness, strength, and impact resistance [5].

The derivation of the macroscopic version of the mechanical energy equation has been scarcely addressed in the literature. Some relevant works are those by Cushman [6], who used the formalism of statistical mechanics to derive the average version of the total energy equation. A more recent derivation is available from the thermodynamically constrained averaging theory (TCAT) [7] by Gray and Miller, where this equation is used together with those from momentum and mass transport in order to derive a constrained entropy inequality, which is a key part of the TCAT theory. Following also a thermodynamic approach, Borja [8] analyzed the mechanical energy equation for unsaturated porous media. Furthermore, Zhu et al. [9], using the volume averaging method [10], showed that the macroscopic form of the mechanical energy equation is equivalent to the result of taking the inner product of the seepage velocity with Darcy's law for single-phase unsteady flow in homogeneous porous media. In their analysis, they found that the term related to the rate of viscous dissipation is equal to Darcy's term dotted with the seepage velocity. Nevertheless, this result may not necessarily hold near the porous medium boundaries, where rapid variations of the effective properties (velocity, permeability, porosity) take place as it is the case in many engineering applications. In addition, if more complicated situations are considered, such as multiphase flow or compressible flow, there are more reasons to doubt that the macroscopic energy equation in porous media is simply the result of the inner product of Darcy's law with the seepage 
velocity. Despite the plausible interest in these and other more complicated situations, the focus in this work is on the derivation of the mechanical energy equation using the method of volume averaging in the case of single-phase and steadystate flow of an incompressible fluid that saturates a rigid porous medium. As a first approach, isothermal conditions are assumed, so only the mechanical part of the total energy equation is justified. Our aim is to first derive an expression for momentum transport that is sufficiently general so it can be applied everywhere in the system, i.e., in the bulk and near the porous medium boundaries, through which momentum exchange can occur (e.g., between a porous medium and a fluid or between two porous media). This expression can be subsequently simplified by imposing pertinent scaling postulates so the Darcy-Brinkman-Forchheimer (DBF) or just the Darcy-Forchheimer (DF) equations can be deduced from this general model. A relevant question to be addressed is if the result of upscaling the pore-scale mechanical energy equation is compatible with the result of taking the inner product of the DBF equation with the seepage velocity. This will allow us to determine up to which point the result from Zhu et al. [9] is valid and to provide a more general form.

The paper is organized as follows: first, we present the governing equations and boundary conditions for mass, momentum, and mechanical energy at the pore scale. The main steps involved in the volume averaging method are then recalled. Since our interest lies in the correspondence between the macroscopic mechanical energy and momentum transport equations (both near the boundaries and in the porous medium bulk) at the Darcy scale, we first direct the attention to the averaging of the momentum transport equation and then apply the volume averaging method to the mechanical energy equation. Finally, the corresponding discussions and conclusions are provided, taking into account extensions to other flow situations.

\section{PORE-SCALE MODEL}

Let us consider the steady, incompressible, and Newtonian flow of a single fluid-phase $\beta$ saturating a rigid and homogeneous porous medium, as shown in Fig. 1. Flow is assumed to take place in a range of the Reynolds number, justifying that both inertial and viscous forces play a significant role and the no-slip boundary condition is assumed to be applicable at the solid-fluid interface. On the basis of these starting assumptions, the governing equations for total mass and momentum in the $\beta$-phase are

$$
\begin{aligned}
\nabla \cdot \mathbf{v}_{\beta} & =0 \\
\rho \mathbf{v}_{\beta} \cdot \nabla \mathbf{v}_{\beta} & =-\nabla p_{\beta}+\rho_{\beta} \mathbf{g}+\mu \nabla^{2} \mathbf{v}_{\beta} .
\end{aligned}
$$

The fluid velocity is subject to the interfacial boundary condition:

$$
\mathbf{v}_{\beta}=\mathbf{0}, \quad \text { at } \mathscr{A}_{\beta \sigma} .
$$

To complete the statement of the boundary-value problem, it is necessary to provide the boundary conditions at entrances

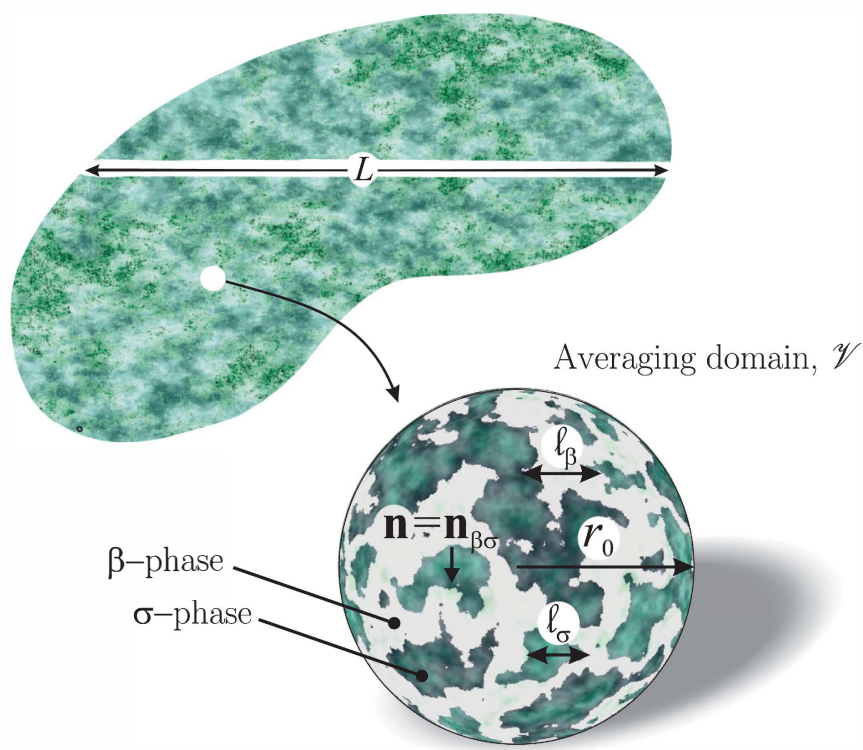

FIG. 1. Sketch of a porous medium including the averaging domain and the characteristic lengths.

and exits of the macroscopic system $\left(\mathscr{A}_{\beta, e}\right)$, which can be written as

$$
\mathbf{v}_{\beta}=\mathbf{v}_{\beta, e}, \quad \text { at } \mathscr{A}_{\beta, e},
$$

where $\mathbf{v}_{\beta, e}$ is assumed to be a known function of position.

The solution of this boundary value problem provides the pressure and velocity fields everywhere in the $\beta$-phase from which one could analyze the different terms in the pore-scale mechanical energy equation that is formally obtained after a dot product of the momentum equation with the velocity, yielding

$$
\begin{aligned}
\frac{\rho}{2} \nabla \cdot\left(v_{\beta}^{2} \mathbf{v}_{\beta}\right)= & -\nabla \cdot\left(p_{\beta} \mathbf{v}_{\beta}\right)+\rho \mathbf{g} \cdot \mathbf{v}_{\beta} \\
& +\frac{1}{2} \mu \nabla^{2} v_{\beta}^{2}-\mu \nabla \mathbf{v}_{\beta}^{T}: \nabla \mathbf{v}_{\beta} .
\end{aligned}
$$

Here, as in the remainder of the development, the nested convention is adopted for the double inner products, i.e., $\mathbf{A}: \mathbf{B}=A_{i j} B_{j i}(=\mathbf{B}: \mathbf{A})$. In addition, fluid density and viscosity are assumed to be constants.

Although achievable, the pointwise detailed solution on $p_{\beta}$ and $\mathbf{v}_{\beta}$ may not be of interest while a macroscopic description is more relevant. As a consequence, there is a necessity for a systematic filtering of nonredundant information arising from the pore-scale model, a procedure that is referred to as upscaling [11]. In this way, it is desirable to derive upscaled models starting from the pore-scale equations by means of a convenient averaging procedure. There are many methodologies to carry out this task such as the method of moments, homogenization, or, more recently, the thermodynamically constrained averaging theory. In this work, the method of volume averaging is employed to derive the upscaled models that are valid both at the porous medium bulk and near its boundaries. Some salient features of this method is that it not only provides the means to derive the upscaled 
models but also allows us to clearly identify the associated scaling postulates involved in the derivation of the models and incorporates a closure scheme to predict the associated macroscopic coefficients appearing in the model [12]. To have a clear view of the above, the main steps of the method of volume averaging are described in the following section.

\section{OVERVIEW OF THE VOLUME AVERAGING METHOD}

In Fig. 2 we show a scheme that describes the method of volume averaging. This scheme should be read from up to down and from left to right. In the latter case, the colors illustrate the systematic loss of redundant information, which we refer to as upscaling. Throughout this work, we will follow Wood [11] and make a distinction between the mathematical operation of averaging and upscaling, because the first one does not require any reduction in the number of degrees of freedom involved in the model. For this reason, Fig. 2 shows two routes that can be followed to obtain different average models. The first route is followed by the continuous lines and it describes the classical method of volume averaging as used in Ref. [10], which leads to a closed upscaled model. The dashed lines in Fig. 2 indicate a second route that leads to an average equation that does not involve upscaling and is known as the one-domain approach [13], which can be further simplified to also yield a closed upscaled model as in the first route. In this work, we will follow this last approach. The rest of this section is devoted to explain in more detail each step represented in Fig. 2.

The first step of the method is the statement of the pore-scale model, which relies on a set of starting assumptions (number 1 in Fig. 2); this step was already described with the associated hypotheses in the previous section. Next, an averaging domain, $\mathscr{V}$ (of norm $V$ ), such as the one shown in Fig. 1, is defined in such a way that it contains portions of all the phases involved in the analysis (i.e., $\mathscr{V}=\mathscr{V}_{\beta}+\mathscr{V}_{\sigma}$ ) (letter a in Fig. 2). In terms of the averaging domain, the superficial and intrinsic averaging operators are introduced for a piecewise function

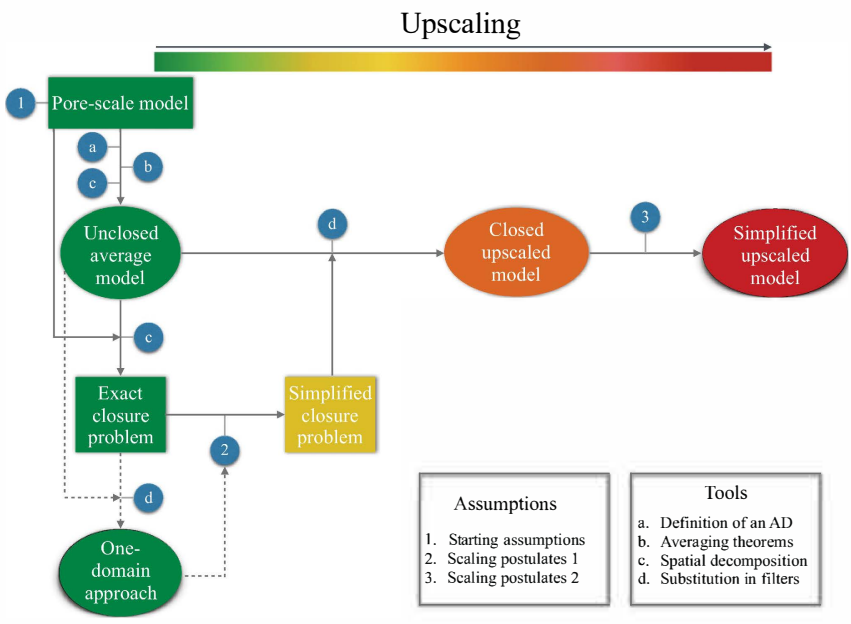

FIG. 2. Diagram of the volume averaging method. The continuous lines and arrows lead to the classical approach whereas the dashed lines and arrows lead to the one-domain approach.

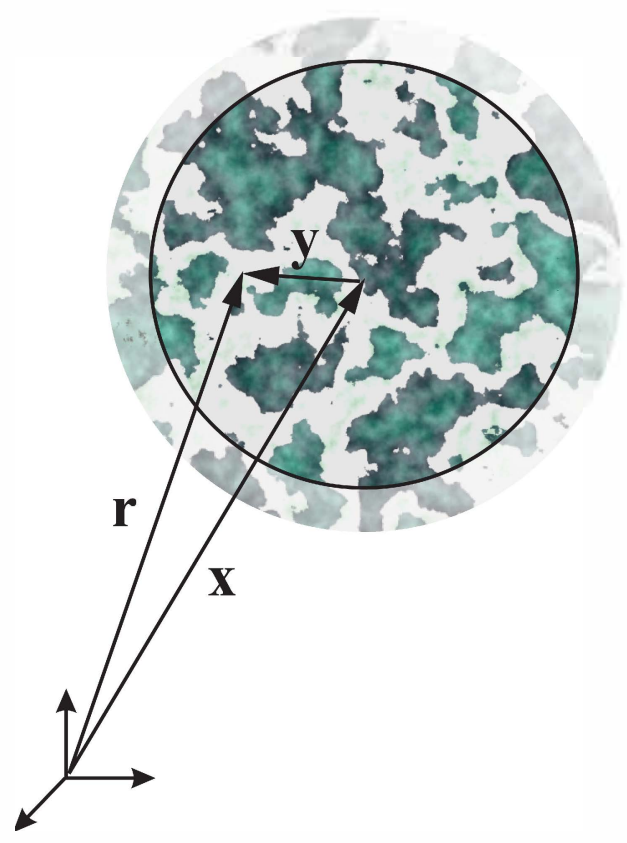

FIG. 3. Position vectors associated to the averaging domain.

defined everywhere in the $\beta$-phase, $\psi_{\beta}$, as follows:

$$
\begin{aligned}
\left.\left\langle\psi_{\beta}\right\rangle\right|_{\mathbf{x}} & =\left.\frac{1}{V} \int_{\mathbf{y} \in \mathscr{V}_{\beta}(\mathbf{x})} \psi_{\beta}\right|_{\mathbf{r}} d V, \\
\left.\left\langle\psi_{\beta}\right\rangle^{\beta}\right|_{\mathbf{x}} & =\left.\frac{1}{V_{\beta}(\mathbf{x})} \int_{\mathbf{y} \in \mathscr{V}_{\beta}(\mathbf{x})} \psi_{\beta}\right|_{\mathbf{r}} d V .
\end{aligned}
$$

Throughout this work, the equations written in terms of the piecewise function $\psi_{\beta}$ are applicable to scalar, vectorial, or tensorial quantities. In the above equations, the vector $\mathbf{x}$ locates the position of the centroid of the averaging domain whereas the position vectors $\mathbf{y}$ and $\mathbf{r}$ locate points in the $\beta$-phase relative to $\mathbf{x}$ and to an arbitrary reference system, respectively, as shown in Fig. 3. The superficial and intrinsic averaging operators are related by the Dupuit-Forchheimer equation:

$$
\left.\left\langle\psi_{\beta}\right\rangle\right|_{\mathbf{x}}=\left.\varepsilon(\mathbf{x})\left\langle\psi_{\beta}\right\rangle^{\beta}\right|_{\mathbf{x}},
$$

where $\varepsilon(\mathbf{x}) \equiv V_{\beta}(\mathbf{x}) / V$ is the volume fraction of the $\beta$-phase contained in the averaging domain.

The superficial averaging operator is subsequently applied to the governing equations at the pore scale. This operation requires interchanging differentiation and integration (letter $\mathrm{b}$ in Fig. 2), which can be achieved by means of the general transport theorem [1],

$$
\left.\left\langle\frac{\partial \psi_{\beta}}{\partial t}\right\rangle\right|_{\mathbf{x}}=\frac{\left.\partial\left\langle\psi_{\beta}\right\rangle\right|_{\mathbf{x}}}{\partial t}-\frac{1}{V} \int_{\mathbf{y} \in \mathscr{A}_{\beta \sigma}(\mathbf{x})} \mathbf{n} \cdot \psi_{\beta} \mathbf{w} d A,
$$

w being the velocity of $\mathscr{A}_{\beta \sigma}$, and the spatial averaging theorem [14]:

$$
\left.\left\langle\nabla \psi_{\beta}\right\rangle\right|_{\mathbf{x}}=\left.\nabla\left\langle\psi_{\beta}\right\rangle\right|_{\mathbf{x}}+\frac{1}{V} \int_{\mathbf{y} \in \mathscr{A}_{\beta \sigma}(\mathbf{x})} \mathbf{n} \psi_{\beta} d A .
$$


For the case of the average of the divergence of a vectorial function, the spatial averaging theorem is slightly modified as indicated in Eq. (1.2-23) in Ref. [10]. It is worth mentioning that the interfacial integrals involved in the above theorems often allow direct substitution of the corresponding boundary conditions. At this point in the analysis, the average model involves integrals of pore-scale quantities and their averaged counterparts. We will refer to the integrals of pore-scale quantities as filters of information as suggested by Whitaker [10]. To eliminate the remaining pore-scale functions, the spatial decomposition introduced by Gray [15] is used (letter c in Fig. 2):

$$
\left.\psi_{\beta}\right|_{\mathbf{r}}=\left.\left\langle\psi_{\beta}\right\rangle^{\beta}\right|_{\mathbf{r}}+\left.\tilde{\psi}_{\beta}\right|_{\mathbf{r}}
$$

For the remaining of this work, we will make a distinction between average quantities evaluated at $\mathbf{r}$ or at $\mathbf{x}$, but we will no longer specify that pore-scale quantities (i.e., $\psi_{\beta}$ and $\tilde{\psi}_{\beta}$ ) are evaluated at $\mathbf{r}$.

In this way, the resulting average model is unclosed because it involves two unknowns (the deviations and average quantities) and only one equation. To overcome this issue, it is necessary to derive and formally solve a boundary-value problem for the deviations fields, which we refer to as a closure problem. This is achieved on the basis of the spatial decomposition given by Eq. (6) and we will illustrate this in the following section. If no assumptions are made, then we can qualify this closure problem to be exact because it contains all the information from the pore scale. As explained by Wood and Valdés-Parada [12], despite the considerable complexity of this problem, it is possible to formally solve it using integral equation formulations based on Green's functions. If this solution is substituted into the filters of the unclosed model (letter $\mathrm{d}$ in Fig. 2), then the resulting average (but not upscaled) equation is the so-called one-domain approach because it can be applied everywhere in the system, i.e., in the bulk of the porous medium and near its boundaries. This completes the nonclassical route of application of the volume averaging method as explained above. Certainly, one may apply scaling postulates in order to obtain simplified versions of the one-domain approach and to even obtain the resulting simplified upscaled model that is only applicable in the bulk of the porous medium.

Analyzing this modeling approach, it is not hard to deduce that the kernel of the upscaling process relies on the systematic reduction of the information contained in the closure problem solution. Therefore, if reasonable scaling postulates (usually in the form of length-scale constraints and assumptions) are adopted (number 2 in Fig. 2), then one may significantly reduce the amount of information carried by the deviations variables and yet still capture the essential (i.e., nonredundant) information from the pore-scale model. The formal solution of this simplified closure problem can be substituted into the filters of the unclosed average model (letter d in Fig. 2) to obtain its closed form. However, the resulting model is still subject to additional simplifications that are based on complementary scaling postulates (number 3 in Fig. 2) in order to obtain a simplified closed upscaled model. In the following section, we will follow the methodology outlined here, i.e., we first derive the one-domain approach models for mass and momentum transport in porous media. Afterwards, pertinent scaling postulates are introduced in order to derive the corresponding models that are valid only in the bulk of the porous medium. Then a similar procedure is applied to the mechanical energy equation.

\section{UPSCALING MASS AND MOMENTUM TRANSPORT}

\section{A. Averaging}

Let us, for the moment, direct the attention to mass transport and apply the superficial averaging operator as defined by Eq. (3a) to Eq. (1a). The resulting equation is

$$
\left.\left\langle\nabla \cdot \mathbf{v}_{\beta}\right\rangle\right|_{\mathbf{x}}=0 .
$$

As explained in the previous section, with the aim of interchanging spatial differentiation and integration, we use the spatial averaging theorem in order to obtain:

$$
\left.\nabla \cdot\left\langle\mathbf{v}_{\beta}\right\rangle\right|_{\mathbf{x}}=0 .
$$

Here we have taken into account the nonslip boundary condition that was imposed at the solid-fluid interface. It should be noted that this result is written in terms of the seepage velocity, which is the common form of averaging for the velocity found in the literature. As will be shown below, for the derivation of the closure problem, it is necessary to express the continuity equation in terms of the intrinsic averaged velocity. This is easily achieved by means of the Dupuit-Forchheimer relation [Eq. (4)]:

$$
\left.\nabla \cdot\left\langle\mathbf{v}_{\beta}\right\rangle^{\beta}\right|_{\mathbf{x}}=-\left.\nabla \ln \varepsilon \cdot\left\langle\mathbf{v}_{\beta}\right\rangle^{\beta}\right|_{\mathbf{x}} \cdot
$$

Directing the attention to momentum transport, let us apply the superficial averaging operator to Eq. (1b), taking into account the continuity equation, to obtain:

$$
\left.\rho\left\langle\nabla \cdot \mathbf{v}_{\beta} \mathbf{v}_{\beta}\right\rangle\right|_{\mathbf{x}}=-\left.\left\langle\nabla p_{\beta}\right\rangle\right|_{\mathbf{x}}-\varepsilon \rho \mathbf{g}+\left.\mu\left\langle\nabla^{2} \mathbf{v}_{\beta}\right\rangle\right|_{\mathbf{x}} \cdot
$$

The result of using the spatial averaging theorem to the transport terms in the above equation is

$$
\begin{aligned}
\left.\rho \boldsymbol{\nabla} \cdot\left\langle\mathbf{v}_{\beta} \mathbf{v}_{\beta}\right\rangle\right|_{\mathbf{x}}= & -\left.\nabla\left\langle p_{\beta}\right\rangle\right|_{\mathbf{x}}+\varepsilon \rho \mathbf{g}+\left.\mu \nabla^{2}\left\langle\mathbf{v}_{\beta}\right\rangle\right|_{\mathbf{x}} \\
& +\frac{1}{V} \int_{\mathbf{y} \in \mathscr{A}_{\beta \sigma}(\mathbf{x})} \mathbf{n} \cdot\left(-\mathbf{I} p_{\beta}+\mu \nabla \mathbf{v}_{\beta}\right) d A
\end{aligned}
$$

or, in terms of intrinsic averages,

$$
\begin{aligned}
\rho \boldsymbol{\nabla} \cdot\left(\left.\varepsilon\left\langle\mathbf{v}_{\beta} \mathbf{v}_{\beta}\right\rangle^{\beta}\right|_{\mathbf{x}}\right)= & -\left.\varepsilon \nabla\left\langle p_{\beta}\right\rangle^{\beta}\right|_{\mathbf{x}}+\varepsilon \rho \mathbf{g}+\left.\mu \varepsilon \nabla^{2}\left\langle\mathbf{v}_{\beta}\right\rangle^{\beta}\right|_{\mathbf{x}} \\
& +\mu \nabla \cdot\left(\left.\nabla \varepsilon\left\langle\mathbf{v}_{\beta}\right\rangle^{\beta}\right|_{\mathbf{x}}\right) \\
& +\frac{1}{V} \int_{\mathbf{y} \in \mathscr{A}_{\mathcal{B}_{\sigma}}(\mathbf{x})} \mathbf{n} \cdot\left[-\mathbf{I}\left(p_{\beta}-\left.\left\langle p_{\beta}\right\rangle^{\beta}\right|_{\mathbf{x}}\right)\right. \\
& \left.+\mu \nabla\left(\mathbf{v}_{\beta}-\left.\left\langle\mathbf{v}_{\beta}\right\rangle^{\beta}\right|_{\mathbf{x}}\right)\right] d A .
\end{aligned}
$$

With the aim of eliminating the pore-scale pressure and velocity contained in the surface and volume integrals (i.e., the filters) from this expression, we use the spatial decomposition defined in Eq. (6) so the result can be written as

$$
\begin{aligned}
& \rho \boldsymbol{\nabla} \cdot\left(\left.\varepsilon\left\langle\left.\left.\left\langle\mathbf{v}_{\beta}\right\rangle^{\beta}\right|_{\mathbf{r}}\left\langle\mathbf{v}_{\beta}\right\rangle^{\beta}\right|_{\mathbf{r}}+\left.\left\langle\mathbf{v}_{\beta}\right\rangle^{\beta}\right|_{\mathbf{r}} \tilde{\mathbf{v}}_{\beta}+\left.\tilde{\mathbf{v}}_{\beta}\left\langle\mathbf{v}_{\beta}\right\rangle^{\beta}\right|_{\mathbf{r}}+\tilde{\mathbf{v}}_{\beta} \tilde{\mathbf{v}}_{\beta}\right\rangle^{\beta}\right|_{\mathbf{x}}\right) \\
& =-\left.\varepsilon \nabla\left\langle p_{\beta}\right\rangle^{\beta}\right|_{\mathbf{x}}+\varepsilon \rho \mathbf{g}+\left.\mu \varepsilon \nabla^{2}\left\langle\mathbf{v}_{\beta}\right\rangle^{\beta}\right|_{\mathbf{x}}+\mu \nabla \cdot\left(\left.\nabla \varepsilon\left\langle\mathbf{v}_{\beta}\right\rangle^{\beta}\right|_{\mathbf{x}}\right)
\end{aligned}
$$




$$
\begin{aligned}
& +\frac{1}{V} \int_{\mathbf{y} \in \mathscr{A}_{\beta \sigma}(\mathbf{x})} \mathbf{n} \cdot\left[-\mathbf{I}\left(\tilde{p}_{\beta}+\Delta\left\langle p_{\beta}\right\rangle^{\beta}\right)\right. \\
& \left.+\mu \nabla\left(\tilde{\mathbf{v}}_{\beta}+\Delta\left\langle\mathbf{v}_{\beta}\right\rangle^{\beta}\right)\right] d A .
\end{aligned}
$$

It should be noted that each term in the above equation is evaluated at $\mathbf{x}$. In addition, in the surface integral of the above equation, we used the following definition:

$$
\Delta\left\langle\psi_{\beta}\right\rangle^{\beta}=\left.\left\langle\psi_{\beta}\right\rangle^{\beta}\right|_{\mathbf{r}}-\left.\left\langle\psi_{\beta}\right\rangle^{\beta}\right|_{\mathbf{x}}, \quad \psi_{\beta}=p_{\beta}, \mathbf{v}_{\beta} .
$$

\section{B. Exact closure problem}

Equation (13) is the unclosed average model. Our goal now is to derive expressions for the velocity and pressure deviations. With this in mind, it is necessary to derive and formally solve the boundary-value problem that governs the fields of $\tilde{p}_{\beta}$ and $\tilde{\mathbf{v}}_{\beta}$. Substitution of Eq. (6) into the pore-scale continuity equation [Eq. (1a)] yields

$$
\boldsymbol{\nabla} \cdot \tilde{\mathbf{v}}_{\beta}=-\boldsymbol{\nabla} \cdot \underbrace{\left\langle\mathbf{v}_{\beta}\right\rangle^{\beta} \mid \mathbf{r}}_{\substack{\text { volumetric } \\ \text { source }}} .
$$

Directing the attention to the momentum transport equation, let us divide Eq. (13) by $\varepsilon$, evaluate the resulting expression at $\mathbf{x}=\mathbf{r}$ and subtract it to Eq. (1b) in order to obtain:

$$
\begin{aligned}
\rho \underbrace{\nabla}_{\text {volumetric source }} \cdot & {\left[\left.\left.\left\langle\mathbf{v}_{\beta}\right\rangle^{\beta}\right|_{\mathbf{r}}\left\langle\mathbf{v}_{\beta}\right\rangle^{\beta}\right|_{\mathbf{r}}\right] } \\
& +\rho \boldsymbol{\nabla} \cdot\left[\left.\left\langle\mathbf{v}_{\beta}\right\rangle^{\beta}\right|_{\mathbf{r}} \tilde{\mathbf{v}}_{\beta}+\left.\tilde{\mathbf{v}}_{\beta}\left\langle\mathbf{v}_{\beta}\right\rangle^{\beta}\right|_{\mathbf{r}}+\tilde{\mathbf{v}}_{\beta} \tilde{\mathbf{v}}_{\beta}\right] \\
& -\varepsilon^{-1} \rho \underbrace{\left(\nabla \cdot\left[\left.\left\langle\left.\left.\left\langle\mathbf{v}_{\beta}\right\rangle^{\beta}\right|_{\mathbf{z}}\left\langle\mathbf{v}_{\beta}\right\rangle^{\beta}\right|_{\mathbf{z}}\right\rangle\right|_{\mathbf{r}}\right]\right.}_{\text {volumetric source }} \\
& \left.+\nabla \cdot\left[\left.\left\langle\left.\left\langle\mathbf{v}_{\beta}\right\rangle^{\beta}\right|_{\mathbf{z}} \tilde{\mathbf{v}}_{\beta}+\left.\tilde{\mathbf{v}}_{\beta}\left\langle\mathbf{v}_{\beta}\right\rangle^{\beta}\right|_{\mathbf{z}}+\tilde{\mathbf{v}}_{\beta} \tilde{\mathbf{v}}_{\beta}\right\rangle\right|_{\mathbf{r}}\right]\right) \\
= & -\nabla \tilde{p}_{\beta}+\mu \nabla^{2} \tilde{\mathbf{v}}_{\beta}-\mu \varepsilon^{-1} \nabla \cdot\left(\left.\nabla \varepsilon\left\langle\mathbf{v}_{\beta}\right\rangle^{\beta}\right|_{\mathbf{r}}\right) \\
& -\frac{1}{V_{\beta}} \int_{\mathbf{z} \in \mathscr{A}_{\beta \sigma}(\mathbf{r})} \mathbf{n} \cdot\left[-\mathbf{I} \tilde{p}_{\beta}+\mu \nabla \tilde{\mathbf{v}}_{\beta}\right] d A \\
& -\frac{1}{V_{\beta}} \int_{\mathbf{z} \in \mathscr{A}_{\beta \sigma}(\mathbf{r})} \mathbf{n} \cdot\left[-\mathbf{I} \Delta\left\langle p_{\beta}\right\rangle^{\beta}+\mu \nabla\left(\Delta\left\langle\mathbf{v}_{\beta}\right\rangle^{\beta}\right)\right] d A .
\end{aligned}
$$

In this last result, we introduced the dummy vector $\mathbf{z}$, in order to make a distinction with the position vector $\mathbf{r}$ in the volumetric and interfacial integrals.

The corresponding boundary conditions for the deviations, which result from substituting the spatial decomposition as given by Eq. (6) into Eqs. (1c) and (1d), are

$$
\begin{aligned}
& \tilde{\mathbf{v}}_{\beta}=-\left.\left\langle\mathbf{v}_{\beta}\right\rangle^{\beta}\right|_{\mathbf{r}}, \quad \text { at } \mathscr{A}_{\beta \sigma}, \\
& \tilde{\mathbf{v}}_{\beta}=\mathbf{v}_{\beta, e}-\left.\left\langle\mathbf{v}_{\beta}\right\rangle^{\beta}\right|_{\mathbf{r}}, \quad \text { at } \mathscr{A}_{\beta, e} .
\end{aligned}
$$

The formal solution of this problem is feasible using integral equations formulations based on Green's functions, as explained in Ref. [12], and it can be expressed as follows:

$$
\begin{aligned}
& \tilde{\mathbf{v}}_{\beta}=-\underbrace{\int_{\mathbf{y} \in \mathscr{V}_{\beta}(\mathbf{x})} \mathbf{G}_{\mathbf{v}} \cdot \mathbf{r}_{\beta} d V}_{\text {Influence of volumetric sources }} \\
& -\underbrace{\left.\underbrace{}_{\mathbf{\mathscr { A } _ { \beta \sigma } ( \mathbf { x } )}} \mathbf{n} \cdot \mu \boldsymbol{\nabla} \mathbf{G}_{\mathbf{v}} \cdot\left\langle\mathbf{v}_{\beta}\right\rangle^{\beta}\right|_{\mathbf{y}} d A}_{\text {Influence of the interfacial source }} \\
& +\underbrace{\int_{\mathbf{y} \in \mathscr{A}_{\beta, e}(\mathbf{x})} \mathbf{n} \cdot \mu \boldsymbol{\nabla} \mathbf{G}_{\mathbf{v}} \cdot\left(\mathbf{v}_{\beta, e}-\left.\left\langle\mathbf{v}_{\beta}\right\rangle^{\beta}\right|_{\mathbf{y}}\right) d A}_{\text {Influence of the entrances and exits }}, \\
& \tilde{p}_{\beta}=-\underbrace{\int_{\mathbf{y} \in \mathscr{V}_{\beta}(\mathbf{x})} \mathbf{g}_{p} \cdot \mathbf{r}_{\beta} d V}_{\text {Influence of volumetric sources }} \\
& -\left.\underbrace{}_{\mathbf{y} \in \mathscr{A}_{\beta \sigma}(\mathbf{x})} \mathbf{n} \cdot \nabla \mathbf{g}_{p} \cdot\left\langle\mathbf{v}_{\beta}\right\rangle^{\beta}\right|_{\mathbf{y}} d A \\
& +\underbrace{\int_{\mathbf{y} \in \mathscr{A}_{\beta, e}(\mathbf{x})} \mathbf{n} \cdot \nabla \mathbf{g}_{p} \cdot\left(\mathbf{v}_{\beta, e}-\left.\left\langle\mathbf{v}_{\beta}\right\rangle^{\beta}\right|_{\mathbf{y}}\right) d A} .
\end{aligned}
$$

In the above equations, $\mathbf{G}_{\mathbf{v}}$ and $\mathbf{g}_{p}$ represent the corresponding Green's functions for the velocity and the pressure, respectively, and $\mathbf{r}_{\beta}$ represents a linear combination of the volumetric source terms identified in the differential equations (15a) and (15b).

\section{One-domain approach}

Now that we have the solution of the closure problem, the average model given by Eq. (13) is complete. For the sake of simplicity in presentation, we introduce the following definition:

$$
\begin{aligned}
\mathbf{f}_{\beta}(\mathbf{x})= & -\rho \boldsymbol{\nabla} \cdot\left(\left.\varepsilon\left\langle\left.\left\langle\mathbf{v}_{\beta}\right\rangle^{\beta}\right|_{\mathbf{r}} \tilde{\mathbf{v}}_{\beta}+\tilde{\mathbf{v}}_{\beta}\left\langle\mathbf{v}_{\beta}\right\rangle^{\beta} \mid \mathbf{r}+\tilde{\mathbf{v}}_{\beta} \tilde{\mathbf{v}}_{\beta}\right\rangle^{\beta}\right|_{\mathbf{x}}\right) \\
& +\frac{1}{V} \int_{\mathbf{y} \in \mathcal{A}_{\beta \sigma}(\mathbf{x})} \mathbf{n} \cdot\left[-\mathbf{I}\left(\tilde{p}_{\beta}+\Delta\left\langle p_{\beta}\right\rangle^{\beta}\right)\right. \\
& \left.+\mu \nabla\left(\tilde{\mathbf{v}}_{\beta}+\Delta\left\langle\mathbf{v}_{\beta}\right\rangle^{\beta}\right)\right] d A
\end{aligned}
$$

which, once inserted into Eq. (13), yields the final form:

$$
\begin{aligned}
\rho \boldsymbol{\nabla} & \left.\cdot\left\langle\left.\left.\left\langle\mathbf{v}_{\beta}\right\rangle^{\beta}\right|_{\mathbf{r}}\left\langle\mathbf{v}_{\beta}\right\rangle^{\beta}\right|_{\mathbf{r}}\right\rangle\right|_{\mathbf{x}} \\
= & -\left.\varepsilon \boldsymbol{\nabla}\left\langle p_{\beta}\right\rangle^{\beta}\right|_{\mathbf{x}}+\varepsilon \rho \mathbf{g}+\left.\mu \nabla^{2}\left\langle\mathbf{v}_{\beta}\right\rangle\right|_{\mathbf{x}} \\
& -\mu \nabla \varepsilon \cdot \nabla\left(\left.\varepsilon^{-1}\left\langle\mathbf{v}_{\beta}\right\rangle\right|_{\mathbf{x}}\right)+\mathbf{f}_{\beta}(\mathbf{x}) .
\end{aligned}
$$

The structure of this equation resembles its pore-scale counterpart, as given by Eq. (1b); however, it is important to clearly identify the physical meaning of each term in the above equation. The term in the left-hand side, although not written in the traditional form, is the macroscopic inertial term due to the macroscopic convective acceleration; the first term 
in the right-hand side corresponds to the macroscopic normal surface force due to the pressure gradient; the next one is due to volume forces in the fluid phase (gravity). The term $\left.\mu \nabla^{2}\left\langle\mathbf{v}_{\beta}\right\rangle\right|_{\mathbf{x}}$ is usually known in the porous media literature as the Brinkman correction term and it partially captures the macroscopic viscous forces. The term $\mu \nabla \varepsilon \cdot \nabla\left(\left.\varepsilon^{-1}\left\langle\mathbf{v}_{\beta}\right\rangle\right|_{\mathbf{x}}\right)$ has been identified as the second Brinkman correction by Ochoa-Tapia and Whitaker [16]. It complements the mapping of the viscous forces by taking into account also the spatial variations of $\varepsilon$. It is worth noting that this term is not present in the Navier-Stokes equations and it is thus a consequence of the averaging process. This characteristic is also shared by the term $\mathbf{f}_{\beta}$ in which all the contributions involving deviations, as defined in Eqs. (6) and (14), are gathered. As it will be shown later, all the terms involving spatial variations of the porosity and macroscopic velocity vanish in the porous medium bulk; however, the same is not true for $\mathbf{f}_{\beta}$ because the deviations of the averages from their pore-scale counterparts are still present in the bulk. As a matter of fact, only in single-phase systems can this term be safely discarded.

It should be noted that, up to this point, no scaling postulates have been introduced for the derivation of the average model, being consistent with the one-domain approach route depicted in Fig. 2. On the one hand, we have gained generality in the sense that the average model is applicable everywhere in the system; on the other hand, the complexity of this model may prevent its use in practical applications. Indeed, it appears to be simpler to perform direct numerical simulations from which the $\mathbf{f}_{\beta}$ term can be computed than using the solutions given in Eqs. (16). Motivated by this, we will systematically adopt scaling postulates with the aim of reducing the complexity of the one-domain approach model in the rest of this section.

\section{Scaling postulates 1 and simplified closure problem}

From the scheme illustrated in Fig. 2, a first set of scaling postulates can be imposed to simplify the closure problem (number 2 in Fig. 2). Therefore, as a first point of simplification, let us constrain the size of the averaging volume, $r_{0}$, to obey the inequality

$$
\ell \ll r_{0} \ll L,
$$

where $\ell$ represents the largest of the characteristic lengths associated to the pore scale while $L$ denotes the smallest of the characteristic lengths associated to the macroscale. In this way, $\ell=\max \left(\ell_{\beta}, \ell_{\sigma}\right)$, where $\ell_{\beta}$ and $\ell_{\sigma}$ denote the characteristic lengths associated to each phase as sketched in Fig. 1. It is worth mentioning that, using a phase-indicator function, it is possible to define additional relevant quantities such as the variance and the autocorrelation function, which allow determining the characteristic length scales in a more precise manner as explained by Wood and Valdés-Parada [12]. The interested reader is referred to this work for further details. Moreover, it is crucial pointing out that if there is no separation of length scales, then it is not possible to define a representative averaging domain and no further simplifications can be applied to the derivations. As explained by Quintard and Whitaker [17], for disordered media it is reasonable to assume that the size of the averaging domain must be several orders of magnitude larger than the characteristic length associated to the pore scale. However, there may be situations, such as fractal media, in which this assumption can fail. The remainder of this work is thus limited to systems in which it is reasonable to assume that the separation of length scales given in (19) is applicable. Under these conditions, we may refer to the averaging domain as a representative elementary volume (REV) [2].

Analyzing Eq. (18), taking into account the definitions given in Eq. (17), we observe that the one-domain approach model contains average quantities evaluated at $\mathbf{r}$ and at $\mathbf{x}$. Actually, one may use Taylor-series expansions in order to express average quantities evaluated at $\mathbf{r}$ only in terms of average quantities evaluated at $\mathbf{x}$ [10], that is,

$$
\begin{aligned}
\left.\left\langle\psi_{\beta}\right\rangle^{\beta}\right|_{\mathbf{r}}= & \left.\left\langle\psi_{\beta}\right\rangle^{\beta}\right|_{\mathbf{x}}+\left.\mathbf{y} \cdot \nabla\left\langle\psi_{\beta}\right\rangle^{\beta}\right|_{\mathbf{x}} \\
& +\frac{1}{2} \mathbf{y} \mathbf{y}:\left.\nabla \nabla\left\langle\psi_{\beta}\right\rangle^{\beta}\right|_{\mathbf{x}}+\cdots .
\end{aligned}
$$

Performing an order-of-magnitude analysis to the second and third terms on the right-hand side of this result leads to

$$
\begin{gathered}
\left.\mathbf{y} \cdot \nabla\left\langle\psi_{\beta}\right\rangle^{\beta}\right|_{\mathbf{x}}=\mathbf{O}\left(\left.\frac{r_{0}}{L_{\left\langle\psi_{\beta}\right\rangle^{\beta}}}\left\langle\psi_{\beta}\right\rangle^{\beta}\right|_{\mathbf{x}}\right), \\
\mathbf{y y}:\left.\nabla \nabla\left\langle\psi_{\beta}\right\rangle^{\beta}\right|_{\mathbf{x}}=\mathbf{O}\left(\left.\frac{r_{0}^{2}}{L_{\nabla\left\langle\psi_{\beta}\right\rangle^{\beta}} L_{\left\langle\psi_{\beta}\right\rangle^{\beta}}}\left\langle\psi_{\beta}\right\rangle^{\beta}\right|_{\mathbf{x}}\right),
\end{gathered}
$$

where $L_{i}$ is the characteristic length associated to the spatial variations of the $i$ quantity $\left(i=\left\langle\psi_{\beta}\right\rangle^{\beta}, \nabla\left\langle\psi_{\beta}\right\rangle^{\beta}\right)$. To avoid oversimplifications for the problem under consideration, we associate the characteristic lengths $L_{\nabla\left\langle\psi_{\beta}\right\rangle^{\beta}}$ and $L_{\left\langle\psi_{\beta}\right\rangle^{\beta}}$ to the width of the transition zone near the porous medium boundary, say, $\delta_{\omega \eta}$. As determined by Valdés-Parada et al. [18], the width of this zone is roughly $20 r_{0}$, indicating that there is at least one order of magnitude of difference between $\delta_{\omega \eta}$ and $r_{0}$. On this basis, we may accept the approximation

$$
\left.\left.\left\langle\psi_{\beta}\right\rangle^{\beta}\right|_{\mathbf{r}} \approx\left\langle\psi_{\beta}\right\rangle^{\beta}\right|_{\mathbf{x}} .
$$

This implies that the average term on the right-hand side of the spatial decomposition given in Eq. (6) can now be evaluated at $\mathbf{x}$. This allows us to consider average quantities as constants within (surface or volumetric) integrals. A direct consequence of this approximation is the following average constraint for the deviations fields:

$$
\left.\left\langle\tilde{\psi}_{\beta}\right\rangle^{\beta}\right|_{\mathbf{x}}=0
$$

Under these conditions, the closure problem can be significantly reduced. The details of this simplification are given in Appendix, and it suffices here to present the simplified closure problem as

$$
\begin{aligned}
\nabla \cdot \tilde{\mathbf{v}}_{\beta}= & 0, \\
\rho \mathbf{v}_{\beta} \cdot \nabla \tilde{\mathbf{v}}_{\beta}= & -\nabla \tilde{p}_{\beta}+\mu \nabla^{2} \tilde{\mathbf{v}}_{\beta} \\
& -\frac{1}{V_{\beta}} \int_{\mathbf{y} \in \mathscr{A}_{\beta \sigma}(\mathbf{x})} \mathbf{n} \cdot\left(-\mathbf{I} \tilde{p}_{\beta}+\mu \nabla \tilde{\mathbf{v}}_{\beta}\right) d A, \\
\tilde{\mathbf{v}}_{\beta}= & -\left.\left\langle\mathbf{v}_{\beta}\right\rangle^{\beta}\right|_{\mathbf{x}}, \quad \text { at } \mathscr{A}_{\beta \sigma} .
\end{aligned}
$$

At this point, we reduce the domain of solution of the closure problem to a periodic unit cell instead of the entire macroscopic domain. Actually, this simplification is more a convenience 
than a necessity and by no means constrains the use of the resulting average model to periodic systems, which are rarely encountered in practice. In this way, at the entrances and exits of the unit cell we can impose the following periodic boundary condition:

$$
\tilde{\psi}_{\beta}(\mathbf{r})=\tilde{\psi}_{\beta}\left(\mathbf{r}+\mathbf{l}_{i}\right) ; \quad \psi_{\beta}=p_{\beta}, \mathbf{v}_{\beta},
$$

where $\mathbf{l}_{i}$ denotes the lattice vectors of the unit cell. Finally, it is worth recalling that the pressure deviations fields are bounded by the integral constraint

$$
\left.\left\langle\tilde{p}_{\beta}\right\rangle^{\beta}\right|_{\mathbf{x}}=0 .
$$

Indeed, the velocity deviations satisfy a similar constraint, but such a constraint is unnecessary to have a well-posed problem in which the only remaining source term can be taken to be constant within the unit cell domain.

The formal solution of this problem can once again be obtained using integral equations formulations based on Green's functions, and for the sake of simplicity in notation, we express the solution as

$$
\begin{aligned}
& \tilde{\mathbf{v}}_{\beta}=\left.\mathbf{B}_{\beta} \cdot\left\langle\mathbf{v}_{\beta}\right\rangle^{\beta}\right|_{\mathbf{x}}, \\
& \tilde{p}_{\beta}=\left.\mu \mathbf{b}_{\beta} \cdot\left\langle\mathbf{v}_{\beta}\right\rangle^{\beta}\right|_{\mathbf{x}} .
\end{aligned}
$$

Notice that this solution is a simplified version of the formal solution given in Eqs. (16); however, since now there are no volumetric sources and the boundary conditions at the entrances and exits are homogeneous, the only remaining source is the one located at the interface. In this way, the so-called closure variables $\mathbf{B}_{\beta}$ and $\mathbf{b}_{\beta}$ can be conceived as integrals of the corresponding Green's functions associated to the velocity and pressure, respectively. These closure variables solve the following boundary-value problem:

$$
\begin{aligned}
\nabla \cdot \mathbf{B}_{\beta}= & \mathbf{0} \\
\frac{\rho}{\mu} \mathbf{v}_{\beta} \cdot \nabla \mathbf{B}_{\beta}= & -\nabla \mathbf{b}_{\beta}+\nabla^{2} \mathbf{B}_{\beta}, \\
& -\frac{1}{V_{\beta}} \int_{\mathbf{y} \in \mathscr{A}_{\beta \sigma}(\mathbf{x})} \mathbf{n} \cdot\left(-\mathbf{l}_{\beta}+\nabla \mathbf{B}_{\beta}\right) d A, \\
\mathbf{B}_{\beta}= & -\mathbf{I}, \quad \text { at } \mathscr{A}_{\beta \sigma}, \\
\psi_{\beta}(\mathbf{r})= & \psi_{\beta}\left(\mathbf{r}+\mathbf{l}_{i}\right) ; \quad \psi_{\beta}=\mathbf{B}_{\beta}, \mathbf{b}_{\beta}, \\
\left.\left\langle\mathbf{b}_{\beta}\right\rangle^{\beta}\right|_{\mathbf{x}}= & \mathbf{0} .
\end{aligned}
$$

Note that these equations correspond to Eqs. (2.22) in Ref. [19].

It is now opportune to return to the average momentum equation by first noticing that, under the assumptions adopted so far, $\mathbf{f}_{\beta}$ can now be reduced to

$$
\begin{aligned}
\mathbf{f}_{\beta}(\mathbf{x})= & -\rho \nabla \cdot\left(\left.\varepsilon\left\langle\tilde{\mathbf{v}}_{\beta} \tilde{\mathbf{v}}_{\beta}\right\rangle^{\beta}\right|_{\mathbf{x}}\right) \\
& +\frac{1}{V} \int_{\mathbf{y} \in \mathscr{A}_{\beta \sigma}(\mathbf{x})} \mathbf{n} \cdot\left[-\mathbf{I} \tilde{p}_{\beta}+\mu \nabla \tilde{\mathbf{v}}_{\beta}\right] d A .
\end{aligned}
$$

Substitution of the formal closure problem solution as given by Eqs. (25) into the above expression and subsequently into the momentum transport equation [Eq. (18)] yields

$$
\begin{aligned}
\frac{\rho}{\varepsilon} \nabla & \cdot\left(\left.\left.\varepsilon\left\langle\mathbf{v}_{\beta}\right\rangle^{\beta}\right|_{\mathbf{x}} \cdot \mathbf{J}_{\beta} \cdot\left\langle\mathbf{v}_{\beta}\right\rangle^{\beta}\right|_{\mathbf{x}}\right) \\
= & -\left.\nabla\left\langle p_{\beta}\right\rangle^{\beta}\right|_{\mathbf{x}}+\rho \mathbf{g}+\left.\frac{\mu}{\varepsilon} \nabla^{2}\left\langle\mathbf{v}_{\beta}\right\rangle\right|_{\mathbf{x}} \\
& -\mu \nabla \ln \varepsilon \cdot \nabla\left(\left.\varepsilon^{-1}\left\langle\mathbf{v}_{\beta}\right\rangle\right|_{\mathbf{x}}\right)-\left.\mathbf{H}_{\beta}^{-1} \cdot\left\langle\mathbf{v}_{\beta}\right\rangle\right|_{\mathbf{x}} .
\end{aligned}
$$

In this result, we introduced the fourth-order tensor $\mathbf{J}_{\beta}$ and the second-order tensor $\mathbf{H}_{\beta}$, which are defined in terms of the closure variables as follows:

$$
\begin{aligned}
\mathbf{J}_{\beta} & =\mathbf{I}+\left.\left\langle\mathbf{B}_{\beta}^{T} \mathbf{B}_{\beta}\right\rangle^{\beta}\right|_{\mathbf{x}}, \\
\varepsilon \mathbf{H}_{\beta}^{-1} & =-\frac{1}{V_{\beta}} \int_{\mathbf{y} \in \mathscr{A}_{\beta \sigma}(\mathbf{x})} \mathbf{n} \cdot\left(-\mathbf{l b}_{\beta}+\nabla \mathbf{B}_{\beta}\right) d A .
\end{aligned}
$$

From a physical viewpoint, the fourth-order tensor $\mathbf{J}_{\beta}$ helps grouping all the macroscopic inertial contributions in a single term. Furthermore, the term $\left.\left\langle\mathbf{B}_{\beta}^{T} \mathbf{B}_{\beta}\right\rangle^{\beta}\right|_{\mathbf{x}}$ is an inertial filter (in the sense of Whitaker [10]) that corrects the traditional inertial term by taking into account the contributions from the pore scale. In addition, the second-order tensor $\mathbf{H}_{\beta}$, which is a stress filter, may be regarded as an apparent permeability tensor that corresponds to a position-dependent Darcy-Forchheimer tensor. The introduction of these effective-medium coefficients is quite convenient because it allows us to separate the macroscopic inertial and viscous contributions in Eq. (28).

Before moving on, it should be noted that the closure problem given by Eqs. (26) is quite complicated because it involves an integrodifferential equation. With the aim of deriving a purely differential closure problem, we make the following changes of variables as suggested by Whitaker [19]:

$$
\begin{aligned}
\mathbf{E}_{\beta} & =\varepsilon^{-1}\left(\mathbf{B}_{\beta}+\mathbf{I}\right) \cdot \mathbf{H}_{\beta}, \\
\mathbf{e}_{\beta} & =\varepsilon^{-1} \mathbf{b}_{\beta} \cdot \mathbf{H}_{\beta} .
\end{aligned}
$$

These variables solve the following differential closure problem:

$$
\begin{aligned}
\nabla \cdot \mathbf{E}_{\beta} & =\mathbf{0}, \\
\frac{\rho}{\mu} \mathbf{v}_{\beta} \cdot \nabla \mathbf{E}_{\beta} & =-\nabla \mathbf{e}_{\beta}+\nabla^{2} \mathbf{E}_{\beta}+\mathbf{I}, \\
\mathbf{E}_{\beta} & =\mathbf{0}, \quad \text { at } \mathscr{A}_{\beta \sigma}, \\
\psi_{\beta}(\mathbf{r}) & =\psi_{\beta}\left(\mathbf{r}+\mathbf{l}_{i}\right) ; \quad \psi_{\beta}=\mathbf{E}_{\beta}, \mathbf{e}_{\beta}, \\
\left.\left\langle\mathbf{e}_{\beta}\right\rangle^{\beta}\right|_{\mathbf{x}} & =\mathbf{0} .
\end{aligned}
$$

From the above, it may appear that the closure problem requires the solution of the pore-scale problem in order to obtain the pointwise velocity that is present in the inertial term of Eq. (31b), making this boundary-value problem unclosed. However, it has been recently shown [20] that this is not the case, because one may use Eqs. (6) and (25a) in order to obtain a closed form of this problem.

In addition, from the constraint that the intrinsic average of the velocity deviations is zero, it follows that

$$
\left.\left\langle\mathbf{E}_{\beta}\right\rangle\right|_{\mathbf{x}}=\mathbf{H}_{\beta} .
$$


This final form of the closure problem was recently solved by Lasseux et al. [21] for several flow angles and Reynolds number values on some model structures. Furthermore, following the derivations shown in Appendix from the study of gas-slip flow in porous media by Lasseux et al. [22], it is not hard to deduce that the $\mathbf{H}_{\beta}$ tensor can be split into a symmetric and a skew-symmetric part, when restricted to the porous medium bulk (i.e., at $\mathbf{x}=\mathbf{x}_{\omega}$ ), as follows:

$$
\begin{aligned}
\mathbf{H}_{\beta}^{T}= & \underbrace{\left.\left\langle\left(\nabla \mathbf{E}_{\beta}\right)^{T 3}: \nabla \mathbf{E}_{\beta}\right\rangle\right|_{\mathbf{x}_{\omega}}}_{\text {symmetric contribution from viscous dissipation }} \\
& +\underbrace{\left.\frac{\rho}{\mu}\left\langle\mathbf{E}_{\beta}^{T} \cdot\left[\nabla \cdot\left(\mathbf{v}_{\beta} \mathbf{E}_{\beta}\right)\right]\right)\right|_{\mathbf{x}_{\omega}}}_{\text {skew-symmetric contribution from inertial effects }} .
\end{aligned}
$$

In the first term on the right-hand side of the above equation the superscript $T 3$ permutes the first and third indices of a third-order tensor, i.e., $\mathbf{A}_{i j k}^{T 3}=A_{k j i}$.

\section{E. Scaling postulates 2 and closed upscaled model}

With the simplifications to the closure problem adopted above, we have reached the reduced solution given by Eqs. (25) and, consequently, the effective-medium coefficients $\mathbf{J}_{\beta}$ and $\mathbf{H}_{\beta}$ can now be readily computed from the knowledge of closure variables fields. Despite the progress gained so far, the one-domain approach, as given in Eq. (28), still involves position-dependent macroscopic coefficients. Nevertheless, the scaling postulates introduced in the previous section have led to a considerable loss of information from the pore scale. Therefore, from the scheme in Fig. 2, we shall refer to this simplified version of the one-domain approach as a closed upscaled model with position-dependent coefficients. Actually, this simplified version of the one-domain approach has been used in the past for the derivation of jump conditions between a porous medium and a fluid under noninertial conditions [23]. However, in practice, it is more common to use effective-medium equations involving constant coefficients and to account for this simplification by introducing appropriate jump conditions. This motivates the introduction of additional scaling postulates that are directed to further simplify the closed upscaled model and, as indicated in Fig. 2, we will refer to the resulting model as a simplified upscaled model.

With the above goal in mind, we first note that Eq. (28) involves three effective-medium coefficients, namely the volumetric fraction of the fluid-phase within the averaging volume, $\varepsilon(\mathbf{x})$, the apparent permeability tensor $\mathbf{H}_{\beta}(\mathbf{x})$, and, finally, the tensor $\mathbf{J}_{\beta}(\mathbf{x})$ that modifies the dyadic product of the average velocities in the inertial term. Outside the transition region that is located near the porous medium boundaries, i.e., in the porous medium bulk, these coefficients are constants and we denote the last position where this assumption is valid as $\mathbf{x}_{\omega}$. In the transition region, we thus propose the following Taylor-series expansion of the coefficients about $\mathbf{x}_{\omega}$ :

$$
\begin{aligned}
\varphi(\mathbf{x})= & \varphi\left(\mathbf{x}_{\omega}\right)+\left(\mathbf{x}-\mathbf{x}_{\omega}\right) \cdot \nabla \varphi\left(\mathbf{x}_{\omega}\right) \\
& +\frac{1}{2}\left(\mathbf{x}-\mathbf{x}_{\omega}\right)\left(\mathbf{x}-\mathbf{x}_{\omega}\right): \nabla \nabla \varphi\left(\mathbf{x}_{\omega}\right)+\cdots,
\end{aligned}
$$

where $\varphi=\varepsilon, \mathbf{H}_{\beta}, \mathbf{J}_{\beta}$. From this expansion, we note that if we truncate it at the first term, all the effective-medium coefficients in Eq. (28) can be replaced by their corresponding values at the porous medium bulk, which are position independent. Consequently, this equation reduces to the DBF equation with the inclusion of the inertial term:

$$
\begin{aligned}
& \rho \nabla \cdot\left(\left.\left.\left\langle\mathbf{v}_{\beta}\right\rangle^{\beta}\right|_{\mathbf{x}} \cdot \mathbf{J}_{\beta}\left(\mathbf{x}_{\omega}\right) \cdot\left\langle\mathbf{v}_{\beta}\right\rangle^{\beta}\right|_{\mathbf{x}}\right) \\
& \quad=-\left.\nabla\left\langle p_{\beta}\right\rangle^{\beta}\right|_{\mathbf{x}}+\rho \mathbf{g}+\left.\mu \nabla^{2}\left\langle\mathbf{v}_{\beta}\right\rangle^{\beta}\right|_{\mathbf{x}}-\left.\mu \mathbf{H}_{\beta}^{-1}\left(\mathbf{x}_{\omega}\right) \cdot\left\langle\mathbf{v}_{\beta}\right\rangle\right|_{\mathbf{x}} .
\end{aligned}
$$

Despite the drastic simplifications that we have imposed in order to obtain this equation, it may still be used near the porous medium boundaries as long as the errors induced by these approximations are compensated. One way to achieve this compensation is by taking more terms in the expansion given in Eq. (33) and another way is by the introduction of appropriate jump boundary conditions, which is the most common approach.

Finally, in the porous medium bulk, the velocity is position invariant and Eq. (34) is reduced to the form of the DarcyForchheimer equation deduced by Whitaker [see Eqs. (2.50) and (4.8) in Ref. 19]:

$$
\left.\left\langle\mathbf{v}_{\beta}\right\rangle\right|_{\mathbf{x}_{\omega}}=-\frac{\mathbf{H}_{\beta}\left(\mathbf{x}_{\omega}\right)}{\mu} \cdot\left(\left.\nabla\left\langle p_{\beta}\right\rangle^{\beta}\right|_{\mathbf{x}_{\omega}}-\rho \mathbf{g}\right) .
$$

The main difference between the simplified upscaled models given in Eqs. (34) and (35) is that the first one may be used near the porous medium boundaries while the latter is constrained to the porous medium bulk. Before moving on, it is pertinent to point out that, even though the macroscopic viscous and inertial terms have been dropped, the pore-scale inertial and viscous forces contributions are still present in the above equation through the Darcy-Forchheimer term $\mathbf{H}_{\beta}$ as it can be deduced from the analysis of Eqs. (31).

Now that we have completed our analysis of the macroscopic momentum transport equation, we are in position to derive the average and upscaled versions of the mechanical energy equation, which is one of the main results of this article. This is the objective of the following section.

\section{UPSCALING THE MECHANICAL ENERGY EQUATION}

As mentioned in the Introduction, one of the objectives of this work is to derive a form of the macroscopic mechanical energy equation that is applicable both in the porous medium bulk and near its boundaries. This analysis raises interesting questions such as is the resulting expression equal to the inner product of the one-domain approach for momentum transport and the Darcy velocity? Is the result reported by Zhu et al. [9] in the bulk applicable? To this end, we will follow a similar structure of developments as the one used in the previous section with the difference that we will not derive the one-domain approach version of the mechanical energy equation. Instead, we will first derive the closed upscaled version of this equation that is applicable at the porous medium boundaries and contrast it with the result of taking the inner product of the seepage velocity with Eq. (34). Finally, we will verify that when the simplified upscaled model for the mechanical energy equation 


\section{MACROSCOPIC MOMENTUM AND MECHANICAL ENERGY . .}

is used in the bulk, the result is the one reported by Zhu et al. [9].

Let us commence our derivations by applying the superficial averaging operator to Eq. (2) to obtain

$$
\begin{aligned}
\left.\frac{\rho}{2}\left\langle\nabla \cdot\left(v_{\beta}^{2} \mathbf{v}_{\beta}\right)\right\rangle\right|_{\mathbf{x}}= & -\left.\left\langle\nabla \cdot\left(p_{\beta} \mathbf{v}_{\beta}\right)\right\rangle\right|_{\mathbf{x}}+\left.\rho \mathbf{g} \cdot\left\langle\mathbf{v}_{\beta}\right\rangle\right|_{\mathbf{x}} \\
& +\left.\frac{1}{2} \mu\left\langle\nabla^{2} v_{\beta}^{2}\right\rangle\right|_{\mathbf{x}}-\left.\mu\left\langle\nabla \mathbf{v}_{\beta}^{T}: \nabla \mathbf{v}_{\beta}\right\rangle\right|_{\mathbf{x}} .
\end{aligned}
$$

Using the spatial averaging theorem and taking into account the nonslip boundary condition, we can interchange spatial integration and differentiation in order to express the above result as follows:

$$
\begin{aligned}
\left.\frac{\rho}{2} \nabla \cdot\left\langle v_{\beta}^{2} \mathbf{v}_{\beta}\right\rangle\right|_{\mathbf{x}}= & -\left.\nabla \cdot\left\langle p_{\beta} \mathbf{v}_{\beta}\right\rangle\right|_{\mathbf{x}}+\left.\rho \mathbf{g} \cdot\left\langle\mathbf{v}_{\beta}\right\rangle\right|_{\mathbf{x}} \\
& +\left.\frac{1}{2} \mu \nabla^{2}\left\langle v_{\beta}^{2}\right\rangle\right|_{\mathbf{x}}-\left.\mu\left\langle\nabla \mathbf{v}_{\beta}^{T}: \nabla \mathbf{v}_{\beta}\right\rangle\right|_{\mathbf{x}},
\end{aligned}
$$

or, after using the Dupuit-Forchheimer relation [Eq. (4)],

$$
\frac{\rho}{2} \nabla \cdot\left(\left.\varepsilon\left\langle v_{\beta}^{2} \mathbf{v}_{\beta}\right\rangle^{\beta}\right|_{\mathbf{x}}\right)=-\nabla \cdot\left(\left.\varepsilon\left\langle p_{\beta} \mathbf{v}_{\beta}\right\rangle^{\beta}\right|_{\mathbf{x}}\right)
$$

$$
+\left.\rho \mathbf{g} \cdot \varepsilon\left\langle\mathbf{v}_{\beta}\right\rangle^{\beta}\right|_{\mathbf{x}}+\frac{1}{2} \mu \nabla^{2}\left(\left.\varepsilon\left\langle v_{\beta}^{2}\right\rangle^{\beta}\right|_{\mathbf{x}}\right)
$$

$$
-\left.\mu \varepsilon\left\langle\nabla \mathbf{v}_{\beta}^{T}: \nabla \mathbf{v}_{\beta}\right\rangle^{\beta}\right|_{\mathbf{x}} \cdot
$$

To make further progress, let us adopt the scaling postulates 1, so the formal closure problem solution given by Eqs. (25) is applicable. Therefore, taking into account the spatial decomposition in Eq. (6) and the changes of variables indicated in Eqs. (30), we can express the pore-scale velocity and pressure as follows:

$$
\begin{aligned}
& \mathbf{v}_{\beta}=\left.\mathbf{D}_{\beta} \cdot\left\langle\mathbf{v}_{\beta}\right\rangle^{\beta}\right|_{\mathbf{x}}, \\
& p_{\beta}=\left.\mu \mathbf{d}_{\beta} \cdot\left\langle\mathbf{v}_{\beta}\right\rangle^{\beta}\right|_{\mathbf{x}}+\left.\left\langle p_{\beta}\right\rangle^{\beta}\right|_{\mathbf{x}},
\end{aligned}
$$

where, for the sake of simplicity, we have introduced the following closure variables:

$$
\begin{aligned}
\mathbf{D}_{\beta} & =\varepsilon \mathbf{E}_{\beta} \cdot \mathbf{H}_{\beta}^{-1}, \\
\mathbf{d}_{\beta} & =\varepsilon \mathbf{e}_{\beta} \cdot \mathbf{H}_{\beta}^{-1} .
\end{aligned}
$$

Using the definitions given in Eqs. (39), it can be easily deduced that

$$
\begin{aligned}
\left.\left\langle v_{\beta}^{2}\right\rangle^{\beta}\right|_{\mathbf{x}} & =\left.\left.\left\langle\mathbf{v}_{\beta}\right\rangle^{\beta}\right|_{\mathbf{x}}\left\langle\mathbf{v}_{\beta}\right\rangle^{\beta}\right|_{\mathbf{x}}:\left.\left\langle\mathbf{D}_{\beta}^{T} \cdot \mathbf{D}_{\beta}\right\rangle^{\beta}\right|_{\mathbf{x}} \\
\left.\left\langle v_{\beta}^{2} \mathbf{v}_{\beta}\right\rangle^{\beta}\right|_{\mathbf{x}} & =\left.\left.\left\langle\mathbf{v}_{\beta}\right\rangle^{\beta}\right|_{\mathbf{x}}\left\langle\mathbf{v}_{\beta}\right\rangle^{\beta}\right|_{\mathbf{x}}:\left.\left.\left\langle\left(\mathbf{D}_{\beta}^{T} \cdot \mathbf{D}_{\beta}\right) \mathbf{D}_{\beta}\right\rangle^{\beta}\right|_{\mathbf{x}} \cdot\left\langle\mathbf{v}_{\beta}\right\rangle^{\beta}\right|_{\mathbf{x}}, \\
\left.\left\langle p_{\beta} \mathbf{v}_{\beta}\right\rangle^{\beta}\right|_{\mathbf{x}} & =\left.\left.\left\langle p_{\beta}\right\rangle^{\beta}\right|_{\mathbf{x}}\left\langle\mathbf{v}_{\beta}\right\rangle^{\beta}\right|_{\mathbf{x}}+\left.\left.\left.\mu\left\langle\mathbf{v}_{\beta}\right\rangle^{\beta}\right|_{\mathbf{x}} \cdot\left\langle\mathbf{d}_{\beta} \mathbf{D}_{\beta}\right\rangle^{\beta}\right|_{\mathbf{x}} \cdot\left\langle\mathbf{v}_{\beta}\right\rangle^{\beta}\right|_{\mathbf{x}}, \\
\left.\left\langle\nabla \mathbf{v}_{\beta}^{T}: \nabla \mathbf{v}_{\beta}\right\rangle^{\beta}\right|_{\mathbf{x}} & =\left.\left.\left\langle\mathbf{v}_{\beta}\right\rangle^{\beta}\right|_{\mathbf{x}}\left\langle\mathbf{v}_{\beta}\right\rangle^{\beta}\right|_{\mathbf{x}}:\left.\left\langle\left(\nabla \mathbf{D}_{\beta}\right)^{T 3}: \nabla \mathbf{D}_{\beta}\right\rangle^{\beta}\right|_{\mathbf{x}}
\end{aligned}
$$

In the last equation, we have taken into account the separation of length scales that allows us to assume that $\left.\nabla\left\langle\mathbf{v}_{\beta}\right\rangle^{\beta}\right|_{\mathbf{x}} \cdot \mathbf{D}_{\beta}^{T} \ll$ $\left.\nabla \mathbf{D}_{\beta} \cdot\left\langle\mathbf{v}_{\beta}\right\rangle^{\beta}\right|_{\mathbf{x}} \cdot$

Substitution of these results into Eq. (38) yields

$$
\begin{aligned}
& \underbrace{\frac{\rho}{2} \boldsymbol{\nabla} \cdot\left(\left.\left.\varepsilon\left\langle\mathbf{v}_{\beta}\right\rangle^{\beta}\right|_{\mathbf{x}}\left\langle\mathbf{v}_{\beta}\right\rangle^{\beta}\right|_{\mathbf{x}}:\left.\left.\left\langle\left(\mathbf{D}_{\beta}^{T} \cdot \mathbf{D}_{\beta}\right) \mathbf{D}_{\beta}\right\rangle^{\beta}\right|_{\mathbf{x}} \cdot\left\langle\mathbf{v}_{\beta}\right\rangle^{\beta}\right|_{\mathbf{x}}\right)}_{\text {rate of transport of kinetic energy by convection }} \\
& =\underbrace{-\boldsymbol{\nabla} \cdot\left(\left.\left.\varepsilon\left\langle p_{\beta}\right\rangle^{\beta}\right|_{\mathbf{x}}\left\langle\mathbf{v}_{\beta}\right\rangle^{\beta}\right|_{\mathbf{x}}\right)}_{\text {rate of pressure work }}+\underbrace{\left.\rho \mathbf{g} \cdot \varepsilon\left\langle\mathbf{v}_{\beta}\right\rangle^{\beta}\right|_{\mathbf{x}}}_{\text {rate of volume-force work }} \\
& \quad \underbrace{-\mu \nabla \cdot\left(\left.\left.\left.\varepsilon\left\langle\mathbf{v}_{\beta}\right\rangle^{\beta}\right|_{\mathbf{x}} \cdot\left\langle\mathbf{d}_{\beta} \mathbf{D}_{\beta}\right\rangle^{\beta}\right|_{\mathbf{x}} \cdot\left\langle\mathbf{v}_{\beta}\right\rangle^{\beta}\right|_{\mathbf{x}}\right)}_{\text {rate of work due to normal stress }}+\underbrace{\frac{1}{2} \mu \nabla^{2}\left(\left.\left.\varepsilon\left\langle\mathbf{v}_{\beta}\right\rangle^{\beta}\right|_{\mathbf{x}}\left\langle\mathbf{v}_{\beta}\right\rangle^{\beta}\right|_{\mathbf{x}}:\left.\left\langle\mathbf{D}_{\beta}^{T} \cdot \mathbf{D}_{\beta}\right\rangle^{\beta}\right|_{\mathbf{x}}\right)}_{\text {rate of work due to shear stress }} \\
& \underbrace{-\left.\left.\mu \varepsilon\left\langle\mathbf{v}_{\beta}\right\rangle^{\beta}\right|_{\mathbf{x}}\left\langle\mathbf{v}_{\beta}\right\rangle^{\beta}\right|_{\mathbf{x}}:\left.\left\langle\left(\nabla \mathbf{D}_{\beta}\right)^{T 3}: \nabla \mathbf{D}_{\beta}\right\rangle^{\beta}\right|_{\mathbf{x}}}_{\text {rate of kinetic energy loss due to viscous dissipation }},
\end{aligned}
$$

where the physical meaning of each term has been clearly identified. As it was done with momentum transport, let us now adopt the second set of scaling postulates, that are focused to the upscaled model. From the previous section, we recall that the truncation of the expansion defined in Eq. (33), at the first term, allows us to approximate all the terms involving integrals of closure variables to their corresponding values in the bulk, i.e., at $\mathbf{x}_{\omega}$. Under these conditions, we may consider the volume fraction as a constant and divide both sides of the above equation by $\varepsilon$ in order to obtain

$$
\begin{aligned}
\frac{\rho}{2} \nabla & \cdot\left(\left.\left.\left\langle\mathbf{v}_{\beta}\right\rangle^{\beta}\right|_{\mathbf{x}}\left\langle\mathbf{v}_{\beta}\right\rangle^{\beta}\right|_{\mathbf{x}}:\left.\left.\left\langle\left(\mathbf{D}_{\beta}^{T} \cdot \mathbf{D}_{\beta}\right) \mathbf{D}_{\beta}\right\rangle^{\beta}\right|_{\mathbf{x}_{\omega}} \cdot\left\langle\mathbf{v}_{\beta}\right\rangle^{\beta}\right|_{\mathbf{x}}\right) \\
= & -\nabla \cdot\left(\left.\left.\left\langle p_{\beta}\right\rangle^{\beta}\right|_{\mathbf{x}}\left\langle\mathbf{v}_{\beta}\right\rangle^{\beta}\right|_{\mathbf{x}}\right)+\left.\rho \mathbf{g} \cdot\left\langle\mathbf{v}_{\beta}\right\rangle^{\beta}\right|_{\mathbf{x}}-\mu \nabla \cdot\left(\left.\left.\left.\left\langle\mathbf{v}_{\beta}\right\rangle^{\beta}\right|_{\mathbf{x}} \cdot\left\langle\mathbf{d}_{\beta} \mathbf{D}_{\beta}\right\rangle^{\beta}\right|_{\mathbf{x}_{\omega}} \cdot\left\langle\mathbf{v}_{\beta}\right\rangle^{\beta}\right|_{\mathbf{x}}\right) \\
& +\frac{1}{2} \mu \nabla^{2}\left(\left.\left.\left\langle\mathbf{v}_{\beta}\right\rangle^{\beta}\right|_{\mathbf{x}}\left\langle\mathbf{v}_{\beta}\right\rangle^{\beta}\right|_{\mathbf{x}}:\left.\left\langle\mathbf{D}_{\beta}^{T} \cdot \mathbf{D}_{\beta}\right\rangle^{\beta}\right|_{\mathbf{x}_{\omega}}\right)-\left.\left.\mu\left\langle\mathbf{v}_{\beta}\right\rangle^{\beta}\right|_{\mathbf{x}}\left\langle\mathbf{v}_{\beta}\right\rangle^{\beta}\right|_{\mathbf{x}}:\left.\left\langle\left(\nabla \mathbf{D}_{\beta}\right)^{T 3}: \nabla \mathbf{D}_{\beta}\right\rangle^{\beta}\right|_{\mathbf{x}_{\omega}} \cdot
\end{aligned}
$$


This equation is clearly not equal to the result of taking the inner product of Eq. (34) with the seepage velocity. Certainly, the above equation is the analog, in terms of mechanical energy, to the DBF equation, i.e., this equation can be used near the porous medium boundaries as along as there is an appropriate boundary condition that compensates the errors induced by the approximations involved in its derivation.

As a final point of analysis, it is necessary to verify if the upscaled model for the mechanical energy equation, when constrained to the porous medium bulk, does agree with Zhu et al.'s [9] result stating that the macroscopic mechanical energy balance is obtained from the dot product of the macroscopic momentum equation with the seepage velocity. With this in mind, let us fix the centroid of the averaging volume to values that lie in the porous medium bulk, i.e., $\mathbf{x}=\mathbf{x}_{\omega}$, so the velocity can be assumed to be a constant, thus:

$$
\begin{aligned}
0= & -\left.\left.\nabla\left\langle p_{\beta}\right\rangle^{\beta}\right|_{\mathbf{x}_{\omega}} \cdot\left\langle\mathbf{v}_{\beta}\right\rangle^{\beta}\right|_{\mathbf{x}_{\omega}}+\left.\rho \mathbf{g} \cdot\left\langle\mathbf{v}_{\beta}\right\rangle^{\beta}\right|_{\mathbf{x}_{\omega}} \\
& -\left.\left.\mu\left\langle\mathbf{v}_{\beta}\right\rangle^{\beta}\right|_{\mathbf{x}_{\omega}}\left\langle\mathbf{v}_{\beta}\right\rangle^{\beta}\right|_{\mathbf{x}_{\omega}}:\left.\left\langle\left(\nabla \mathbf{D}_{\beta}\right)^{T 3}: \nabla \mathbf{D}_{\beta}\right\rangle^{\beta}\right|_{\mathbf{x}_{\omega}},
\end{aligned}
$$

or, after a little rearrangement,

$$
\begin{aligned}
0= & \left(-\left.\nabla\left\langle p_{\beta}\right\rangle^{\beta}\right|_{\mathbf{x}_{\omega}}+\rho \mathbf{g}\right. \\
& \left.-\left.\left.\mu\left\langle\mathbf{v}_{\beta}\right\rangle^{\beta}\right|_{\mathbf{x}_{\omega}} \cdot\left\langle\left(\nabla \mathbf{D}_{\beta}\right)^{T 3}: \nabla \mathbf{D}_{\beta}\right\rangle^{\beta}\right|_{\mathbf{x}_{\omega}}\right)\left.\cdot\left\langle\mathbf{v}_{\beta}\right\rangle^{\beta}\right|_{\mathbf{x}_{\omega}} .
\end{aligned}
$$

Directing the attention to the last integral term of the above equation and substituting the definitions given in Eqs. (40), one obtains:

$$
\begin{aligned}
& \left.\left\langle\left(\boldsymbol{\nabla} \mathbf{D}_{\beta}\right)^{T 3}: \boldsymbol{\nabla} \mathbf{D}_{\beta}\right\rangle^{\beta}\right|_{\mathbf{x}_{\omega}} \\
& \quad=\left.\varepsilon\left(\mathbf{H}_{\beta}^{-1}\right)^{T} \cdot\left\langle\left(\nabla \mathbf{E}_{\beta}\right)^{T 3}: \nabla \mathbf{E}_{\beta}\right\rangle\right|_{\mathbf{x}_{\omega}} \cdot \mathbf{H}_{\beta}^{-1},
\end{aligned}
$$

or, using Eq. (32),

$$
\begin{aligned}
& \left.\left\langle\left(\boldsymbol{\nabla} \mathbf{D}_{\beta}\right)^{T 3}: \nabla \mathbf{D}_{\beta}\right\rangle^{\beta}\right|_{\mathbf{x}_{\omega}} \\
& \quad=\varepsilon \mathbf{H}_{\beta}^{-1}-\left.\varepsilon\left(\mathbf{H}_{\beta}^{-1}\right)^{T} \cdot \frac{\rho}{\mu}\left\langle\mathbf{E}_{\beta}^{T} \cdot\left[\nabla \cdot\left(\mathbf{v}_{\beta} \mathbf{E}_{\beta}\right)\right]\right\rangle\right|_{\mathbf{x}_{\omega}} \cdot \mathbf{H}_{\beta}^{-1} .
\end{aligned}
$$

Substitution of Eq. (47) into Eq. (45) leads to

$$
\begin{aligned}
0= & \left.\left(-\left.\nabla\left\langle p_{\beta}\right\rangle^{\beta}\right|_{\mathbf{x}_{\omega}}+\rho \mathbf{g}-\left.\mu \varepsilon\left\langle\mathbf{v}_{\beta}\right\rangle^{\beta}\right|_{\mathbf{x}_{\omega}} \cdot \mathbf{H}_{\beta}^{-1}\right) \cdot\left\langle\mathbf{v}_{\beta}\right\rangle^{\beta}\right|_{\mathbf{x}_{\omega}} \\
& +\rho \varepsilon\left(\mathbf{s}_{\beta} \mathbf{s}_{\beta}:\left.\left\langle\mathbf{E}_{\beta}^{T} \cdot\left[\boldsymbol{\nabla} \cdot\left(\mathbf{v}_{\beta} \mathbf{E}_{\beta}\right)\right]\right\rangle\right|_{\mathbf{x}_{\omega}}\right),
\end{aligned}
$$

where, for the sake of brevity in notation, we introduced:

$$
\mathbf{s}_{\beta}=\left.\mathbf{H}_{\beta}^{-1} \cdot\left\langle\mathbf{v}_{\beta}\right\rangle^{\beta}\right|_{\mathbf{x}_{\omega}} .
$$

As indicated in Eq. (32), the second-rank tensor $\left.\left\langle\mathbf{E}_{\beta}^{T} \cdot\left[\nabla \cdot\left(\mathbf{v}_{\beta} \mathbf{E}_{\beta}\right)\right]\right\rangle\right|_{\mathbf{x}_{\omega}}$ is skew-symmetric and, since the dyad $\mathbf{S}_{\beta} \mathbf{S}_{\beta}$ is a symmetric second-order tensor, we can readily conclude that the last term on the right-hand side of Eq. (48) is zero. After multiplication by the porous medium porosity, this equation is simplified to

$$
0=\left.\left(-\left.\nabla\left\langle p_{\beta}\right\rangle^{\beta}\right|_{\mathbf{x}_{\omega}}+\rho \mathbf{g}-\left.\mu\left\langle\mathbf{v}_{\beta}\right\rangle\right|_{\mathbf{x}_{\omega}} \cdot \mathbf{H}_{\beta}^{-1}\right) \cdot\left\langle\mathbf{v}_{\beta}\right\rangle\right|_{\mathbf{x}_{\omega}},
$$

which clearly is the inner product of Darcy's law with the seepage velocity, thus verifying the result from Zhu et al. [9] in the porous medium bulk.

\section{DISCUSSIONS AND CONCLUSIONS}

In this work, we addressed the question about the correspondence between the upscaled version of the mechanical energy equation and the inner product of the macroscopic momentum balance equation with the seepage velocity. The following points of discussion and conclusions are hence in order:

(i) Using the method of volume averaging, without the imposition of any length-scale constraints or assumptions, we derived an average model for momentum transport [Eq. (18)]. This one-domain approach, which is valid everywhere in the system (i.e., near the porous medium bulk and its boundaries), was the cornerstone for the derivation of upscaled models for momentum transport. By imposing a first set of scaling postulates, the one-domain approach gave rise to an upscaled model in which the effective-medium coefficients can be readily computed from the solution of ancillary closure problems in representative unit cells. Then a second set of more severe scaling postulates was imposed that lead to a version of the DBF equation that includes inertial contributions [Eq. (34)]. Finally, in the porous medium bulk, this last model reduces to the Darcy-Forchheimer model [Eq. (35)]. The approach for the derivation of these last two upscaled models differs from the classical applications of the volume averaging method reported by Whitaker [19].

(ii) Traditionally, Brinkman's correction to Darcy's law is regarded as an ad hoc addition to Darcy's law that allows the velocity to change with position near the porous medium boundaries, while keeping the effective medium coefficients constant. Actually, this is a contradiction, because, in general, the velocity and the effective-medium coefficients are both functions of position near the porous medium boundaries. This controversy can be solved by the inclusion of the second set of scaling postulates that give rise to the DBF model. Notice that, contrary to Whitaker [19], we have not imposed additional constraints dealing with the Reynolds number, so Eq. (34) maintains the macroscopic inertial contribution term.

(iii) The upscaled model for the mechanical energy equation resulting from applying the volume averaging method [Eq. (43)] was found not to be the result of taking the inner product of the DBF equation with the seepage velocity. However this expression of the macroscopic mechanical energy equation shares the same limitations (in terms of upscaling) as the DBF transport model. To the best of our knowledge, this complete form of the mechanical energy equation has not been previously reported in the literature. If this equation is to be used to compute the power required to reach a certain fluid flow near porous media boundaries through which momentum transport can occur, then it is necessary to acknowledge that the errors that are involved in its derivation should be compensated. There are at least two routes for achieving this goal: one way is to relax the scaling postulates involved in its derivation. For example, one may take more terms in the expansion of effective-medium properties defined in Eq. (33). An alternative is to derive the corresponding jump boundary conditions that incorporate coefficients that account for the information that was lost in the upscaled transport model.

(iv) Finally, in the porous medium bulk, it is not necessary to average the mechanical energy equation at the pore scale, 
because the upscaled model is simply the result of taking the inner product between the Darcy equation with the seepage velocity as shown in Eq. (50). This result may have not been achieved without knowledge of the symmetric and skew-symmetric parts of the apparent permeability tensor $\mathbf{H}_{\beta}$, as indicated in Eq. (32). Our analysis complements and verifies the result from Zhu et al. [9].

As mentioned in the Introduction, the derivation in this work can surely be extended to other more complicated situations such as multiphase flow, compressible flow, and even to nonisothermal flow in porous media. As a final note, it is worth mentioning that the framework used in the present analysis, consisting of the derivation of a one-domain approach, which may be further simplified by the imposition of scaling postulates, is indeed extensible to other practical applications beyond porous media systems. Actually, the main message from this work is that the analysis in terms of energy balance near the boundaries of multiscale systems is incomplete if it deals only with the study of mass and momentum transport, because it is necessary to also consider the upscaled mechanical energy equation.

\section{ACKNOWLEDGMENTS}

C.T.P.G. is thankful to Universidad Autónoma Metropolitana for the scholarship received. F.J.V.P. is thankful to CNRS for the facilities provided to carry out a research internship with D.L. D.L. acknowledges financial support from CONACyT (Grant No. 25623) for a stay in UAM-Iztapalapa to work with F.J.V.P. during autumn 2016.

\section{APPENDIX: SIMPLIFICATIONS OF THE CLOSURE PROBLEM}

Our goal in this section is to detail the simplifications that are applicable to the closure problem associated to the onedomain approach. On the basis of the separation of scales given in (19) and the corollaries presented in Sec. IV, the following simplifications are applicable to Eq. (15b):

(a) The last term on the left-hand side is reduced to

$$
\begin{aligned}
& \left.\nabla \cdot\left\langle\left.\left.\left\langle\mathbf{v}_{\beta}\right\rangle^{\beta}\right|_{\mathbf{z}}\left\langle\mathbf{v}_{\beta}\right\rangle^{\beta}\right|_{\mathbf{z}}+\left.\left\langle\mathbf{v}_{\beta}\right\rangle^{\beta}\right|_{\mathbf{z}} \tilde{\mathbf{v}}_{\beta}+\left.\tilde{\mathbf{v}}_{\beta}\left\langle\mathbf{v}_{\beta}\right\rangle^{\beta}\right|_{\mathbf{z}}+\tilde{\mathbf{v}}_{\beta} \tilde{\mathbf{v}}_{\beta}\right\rangle\right|_{\mathbf{r}} \\
& \approx \nabla \cdot\left(\left.\left.\varepsilon\left\langle\mathbf{v}_{\beta}\right\rangle^{\beta}\right|_{\mathbf{x}}\left\langle\mathbf{v}_{\beta}\right\rangle^{\beta}\right|_{\mathbf{x}}\right)+\left.\nabla \cdot\left\langle\tilde{\mathbf{v}}_{\beta} \tilde{\mathbf{v}}_{\beta}\right\rangle\right|_{\mathbf{x}} .
\end{aligned}
$$

(b) The last term on the right-hand side of this equation vanishes.

In this way, Eq. (15b) reduces to

$$
\begin{aligned}
& \rho \nabla \cdot\left[\left.\left\langle\mathbf{v}_{\beta}\right\rangle^{\beta}\right|_{\mathbf{x}} \tilde{\mathbf{v}}_{\beta}+\left.\tilde{\mathbf{v}}_{\beta}\left\langle\mathbf{v}_{\beta}\right\rangle^{\beta}\right|_{\mathbf{x}}+\tilde{\mathbf{v}}_{\beta} \tilde{\mathbf{v}}_{\beta}\right] \\
& -\left.\left.\rho \nabla \ln \varepsilon \cdot\left\langle\mathbf{v}_{\beta}\right\rangle^{\beta}\right|_{\mathbf{x}}\left\langle\mathbf{v}_{\beta}\right\rangle^{\beta}\right|_{\mathbf{x}}-\left.\varepsilon^{-1} \rho \boldsymbol{\nabla} \cdot\left\langle\tilde{\mathbf{v}}_{\beta} \tilde{\mathbf{v}}_{\beta}\right\rangle\right|_{\mathbf{x}} \\
& =-\nabla \tilde{p}_{\beta}+\mu \nabla^{2} \tilde{\mathbf{v}}_{\beta}-\left.\frac{\mu}{\varepsilon} \nabla^{2} \varepsilon\left\langle\mathbf{v}_{\beta}\right\rangle^{\beta}\right|_{\mathbf{x}}-\left.\mu \nabla \ln \varepsilon \cdot \nabla\left\langle\mathbf{v}_{\beta}\right\rangle^{\beta}\right|_{\mathbf{x}} \\
& -\frac{1}{V_{\beta}} \int_{\mathbf{y} \in \mathscr{A}_{\beta \sigma}(\mathbf{x})} \mathbf{n} \cdot\left(-\mathbf{I} \tilde{p}_{\beta}+\mu \nabla \tilde{\mathbf{v}}_{\beta}\right) d A .
\end{aligned}
$$

To further simplify this equation, we use orders-of-magnitude estimates of the several terms that it contains. In performing this analysis, we take into account the interfacial boundary condition given by Eq. (15c), which indicates that the order of magnitude of the velocity deviations can be taken to be the same as the one for the intrinsic average velocity. Under these conditions, we have the following estimates:

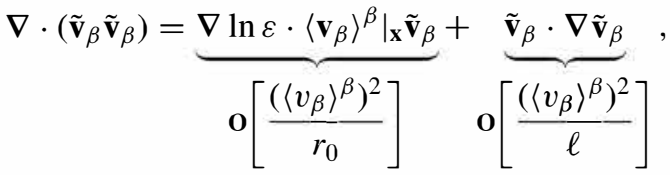

where we have taken $r_{0}$ to be a reasonable estimate of the characteristic length scale of the spatial variations of the porosity. Since we have accepted the separation of length scales $\ell \ll r_{0}$, the above expression can be reduced to

$$
\boldsymbol{\nabla} \cdot\left(\tilde{\mathbf{v}}_{\beta} \tilde{\mathbf{v}}_{\beta}\right)=O\left[\frac{\left(\left\langle v_{\beta}\right\rangle^{\beta}\right)^{2}}{\ell}\right] .
$$

In a similar way, we have the following order-of-magnitude estimates:

$$
\begin{aligned}
\left.\left.\nabla \ln \varepsilon \cdot\left\langle\mathbf{v}_{\beta}\right\rangle^{\beta}\right|_{\mathbf{x}}\left\langle\mathbf{v}_{\beta}\right\rangle^{\beta}\right|_{\mathbf{x}} & =O\left[\frac{\left(\left\langle v_{\beta}\right\rangle^{\beta}\right)^{2}}{r_{0}}\right], \\
\left.\varepsilon^{-1} \nabla \cdot\left\langle\tilde{\mathbf{v}}_{\beta} \tilde{\mathbf{v}}_{\beta}\right\rangle\right|_{\mathbf{x}} & =O\left[\frac{\left(\left\langle v_{\beta}\right\rangle^{\beta}\right)^{2}}{\delta_{\omega \eta}}\right], \\
\nabla^{2} \tilde{\mathbf{v}}_{\beta} & =O\left(\frac{\left\langle v_{\beta}\right\rangle^{\beta}}{\ell^{2}}\right), \\
\left.\frac{1}{\varepsilon} \nabla^{2} \varepsilon\left\langle\mathbf{v}_{\beta}\right\rangle^{\beta}\right|_{\mathbf{x}} & =O\left(\frac{\left\langle v_{\beta}\right\rangle^{\beta}}{r_{0}^{2}}\right), \\
\left.\nabla \ln \varepsilon \cdot \nabla\left\langle\mathbf{v}_{\beta}\right\rangle^{\beta}\right|_{\mathbf{x}} & =O\left(\frac{\left\langle v_{\beta}\right\rangle^{\beta}}{r_{0} \delta_{\omega \eta}}\right) .
\end{aligned}
$$

In this way, on the basis of the length-scale constraints,

$$
\ell \ll r_{0} ; \quad \ell \ll \delta_{\omega \eta},
$$

it is reasonable to assume that

$$
\begin{aligned}
\left.\left.\nabla \ln \varepsilon \cdot\left\langle\mathbf{v}_{\beta}\right\rangle^{\beta}\right|_{\mathbf{x}}\left\langle\mathbf{v}_{\beta}\right\rangle^{\beta}\right|_{\mathbf{x}} & \ll \nabla \cdot\left(\tilde{\mathbf{v}}_{\beta} \tilde{\mathbf{v}}_{\beta}\right), \\
\left.\varepsilon^{-1} \nabla \cdot\left\langle\tilde{\mathbf{v}}_{\beta} \tilde{\mathbf{v}}_{\beta}\right\rangle\right|_{\mathbf{x}} & \ll \nabla \cdot\left(\tilde{\mathbf{v}}_{\beta} \tilde{\mathbf{v}}_{\beta}\right), \\
\left.\frac{1}{\varepsilon} \nabla^{2} \varepsilon\left\langle\mathbf{v}_{\beta}\right\rangle^{\beta}\right|_{\mathbf{x}} & \ll \nabla^{2} \tilde{\mathbf{v}}_{\beta}, \\
\left.\nabla \ln \varepsilon \cdot \nabla\left\langle\mathbf{v}_{\beta}\right\rangle^{\beta}\right|_{\mathbf{x}} & \ll \nabla^{2} \tilde{\mathbf{v}}_{\beta} .
\end{aligned}
$$

Under these conditions, we can reduce Eq. (A1) to

$$
\begin{aligned}
& \rho \nabla \cdot\left[\left.\left\langle\mathbf{v}_{\beta}\right\rangle^{\beta}\right|_{\mathbf{x}} \tilde{\mathbf{v}}_{\beta}+\left.\tilde{\mathbf{v}}_{\beta}\left\langle\mathbf{v}_{\beta}\right\rangle^{\beta}\right|_{\mathbf{x}}+\tilde{\mathbf{v}}_{\beta} \tilde{\mathbf{v}}_{\beta}\right] \\
& =-\nabla \tilde{p}_{\beta}+\mu \nabla^{2} \tilde{\mathbf{v}}_{\beta}-\frac{1}{V_{\beta}} \int_{\mathbf{y} \in \mathscr{A}_{\beta \sigma}(\mathbf{x})} \mathbf{n} \cdot\left(-\mathbf{I} \tilde{p}_{\beta}+\mu \nabla \tilde{\mathbf{v}}_{\beta}\right) d A .
\end{aligned}
$$

Directing the attention to the left-hand side term of the above equation, we have

$$
\begin{aligned}
& \boldsymbol{\nabla} \cdot\left[\left.\left\langle\mathbf{v}_{\beta}\right\rangle^{\beta}\right|_{\mathbf{x}} \tilde{\mathbf{v}}_{\beta}+\left.\tilde{\mathbf{v}}_{\beta}\left\langle\mathbf{v}_{\beta}\right\rangle^{\beta}\right|_{\mathbf{x}}+\tilde{\mathbf{v}}_{\beta} \tilde{\mathbf{v}}_{\beta}\right] \\
& \quad=\left.\left.\nabla \ln \varepsilon \cdot\left\langle\mathbf{v}_{\beta}\right\rangle^{\beta}\right|_{\mathbf{x}}\left\langle\mathbf{v}_{\beta}\right\rangle^{\beta}\right|_{\mathbf{x}}+\left.\tilde{\mathbf{v}}_{\beta} \cdot \nabla\left\langle\mathbf{v}_{\beta}\right\rangle^{\beta}\right|_{\mathbf{x}}+\mathbf{v}_{\beta} \cdot \nabla \tilde{\mathbf{v}}_{\beta} .
\end{aligned}
$$


Once again, with the intention of simplifying this result, we make use of the following orders-of-magnitude estimates:

$$
\begin{aligned}
\left.\left.\nabla \ln \varepsilon \cdot\left\langle\mathbf{v}_{\beta}\right\rangle^{\beta}\right|_{\mathbf{x}}\left\langle\mathbf{v}_{\beta}\right\rangle^{\beta}\right|_{\mathbf{x}} & =O\left[\frac{\left(\left\langle v_{\beta}\right\rangle^{\beta}\right)^{2}}{r_{0}}\right], \\
\left.\tilde{\mathbf{v}}_{\beta} \cdot \nabla\left\langle\mathbf{v}_{\beta}\right\rangle^{\beta}\right|_{\mathbf{x}} & =O\left[\frac{\left(\left\langle v_{\beta}\right\rangle^{\beta}\right)^{2}}{\delta_{\omega \eta}}\right], \\
\mathbf{v}_{\beta} \cdot \nabla \tilde{\mathbf{v}}_{\beta} & =O\left[\frac{\left(\left\langle v_{\beta}\right\rangle^{\beta}\right)^{2}}{\ell}\right] .
\end{aligned}
$$

Therefore, on the basis of the length-scale constraints given in (A4), we may assume that

$$
\begin{array}{r}
\left.\left.\nabla \ln \varepsilon \cdot\left\langle\mathbf{v}_{\beta}\right\rangle^{\beta}\right|_{\mathbf{x}}\left\langle\mathbf{v}_{\beta}\right\rangle^{\beta}\right|_{\mathbf{x}} \ll \mathbf{v}_{\beta} \cdot \nabla \tilde{\mathbf{v}}_{\beta}, \\
\left.\tilde{\mathbf{v}}_{\beta} \cdot \nabla\left\langle\mathbf{v}_{\beta}\right\rangle^{\beta}\right|_{\mathbf{x}} \ll \mathbf{v}_{\beta} \cdot \nabla \tilde{\mathbf{v}}_{\beta} .
\end{array}
$$

Under these conditions, Eq. (A6) reduces to its final form:

$$
\begin{aligned}
\rho \mathbf{v}_{\beta} \cdot \nabla \tilde{\mathbf{v}}_{\beta}= & -\nabla \tilde{p}_{\beta}+\mu \nabla^{2} \tilde{\mathbf{v}}_{\beta} \\
& -\frac{1}{V_{\beta}} \int_{\mathbf{y} \in \mathscr{A}_{\beta \sigma}(\mathbf{x})} \mathbf{n} \cdot\left(-\mathbf{l} \tilde{p}_{\beta}+\mu \nabla \tilde{\mathbf{v}}_{\beta}\right) d A .
\end{aligned}
$$

It is worth noting that this equation no longer contains volumetric sources. In fact, the only volumetric source that remains in the closure problem is given in the right-hand side of Eq. (15a). The order of magnitude of this source is

$$
\left.\frac{1}{V} \int_{\mathbf{y} \in \mathscr{V}_{\beta}(\mathbf{x})} \nabla \ln \varepsilon \cdot\left\langle\mathbf{v}_{\beta}\right\rangle^{\beta}\right|_{\mathbf{x}} d V=O\left(\frac{\left\langle v_{\beta}\right\rangle^{\beta}}{r_{0}}\right) .
$$

This estimate can be contrasted with the order of magnitude of the interfacial source, which is

$$
\left.\frac{1}{V} \int_{\mathbf{y} \in \mathscr{A}_{\beta \sigma}(\mathbf{x})}\left\langle\mathbf{v}_{\beta}\right\rangle^{\beta}\right|_{\mathbf{x}} d A=O\left(\frac{\left\langle v_{\beta}\right\rangle^{\beta}}{\ell}\right) .
$$

From these estimates, we may assume that, due to the lengthscale constraint $\ell \ll r_{0}$, the interfacial source is much larger than the volumetric source. Under these conditions, the closure problem can be expressed as stated in Eqs. (24) in the main text.
[1] R. Bird, W. Stewart, and E. Lightfoot, Transport Phenomena (John Wiley, New York, 2007).

[2] J. Bear and A. Cheng, Modeling Groundwater Flow and Contaminant Transport (Springer, Berlin, 2010).

[3] J. Nordbotten and M. Celia, Geological Storage of $\mathrm{CO}_{2}$ : Modeling Approaches for Large-Scale Simulation (Wiley, New York, 2012).

[4] W. Chen, X. Tan, H. Yu, G. Wu, and S. Jia, J. Rock Mech. Geotech. Eng. 1, 31 (2009).

[5] Z. Xu and M. J. Buehler, Phys. Rev. E 81, 061910 (2010).

[6] J. Cushman, The Physics of Fluids in Hierarchical Porous Media: Angstroms to Miles (Springer, Berlin, 2010).

[7] W. Gray and C. Miller, Introduction to the Thermodynamically Constrained Averaging Theory for Porous Medium Systems, Advances in Geophysical and Environmental Mechanics and Mathematics (Springer, Berlin, 2014).

[8] R. Borja, Int. J. Solids Struct. 43, 1764 (2006).

[9] T. Zhu, C. Waluga, B. Wohlmuth, and M. Manhart, Transp. Porous Media 104, 161 (2014).

[10] S. Whitaker, The Method of Volume Averaging (Kluwer, Amsterdam, 1999).
[11] B. Wood, Adv. Water Res. 32, 723 (2009).

[12] B. Wood and F. Valdés-Parada, Adv. Water Res. 51, 139 (2013).

[13] B. Goyeau, D. Lhuillier, D. Gobin, and M. Velarde, Int. J. Heat Mass Transf. 46, 4071 (2003).

[14] F. Howes and S. Whitaker, Chem. Eng. Sci. 40, 1387 (1985).

[15] W. Gray, Chem. Eng. Sci. 30, 229 (1975).

[16] J. Ochoa-Tapia and S. Whitaker, Int. J. Heat Mass Transf. 38, 2635 (1995).

[17] M. Quintard and S. Whitaker, Transp. Porous Media 14, 163 (1994).

[18] F. Valdés-Parada, C. Aguilar-Madera, J. Ochoa-Tapia, and B. Goyeau, Adv. Water Res. 62, 327 (2013).

[19] S. Whitaker, Transp. Porous Media 25, 27 (1996).

[20] F. Valdés-Parada, D. Lasseux, and F. Bellet, Adv. Water Res. 90, 70 (2016).

[21] D. Lasseux, A. A. Abbasian-Arani, and A. Ahmadi, Phys. Fluids 23, 073103 (2011).

[22] D. Lasseux, F. Valdés-Parada, and M. Porter, J. Fluid Mech. 805, 118 (2016).

[23] F. Valdés-Parada, J. Alvarez-Ramírez, B. Goyeau, and J. OchoaTapia, Transp. Porous Media 78, 439 (2009). 